\title{
Effective Loop Fusion in Polyhedral Compilation Using Fusion Conflict Graphs
}

\author{
ARAVIND ACHARYA and UDAY BONDHUGULA, Indian Institute of Science, India \\ ALBERT COHEN, Google, France
}

Polyhedral auto-transformation frameworks are known to find efficient loop transformations that maximize locality and parallelism and minimize synchronization. While complex loop transformations are routinely modeled in these frameworks, they tend to rely on ad hoc heuristics for loop fusion. Although there exist multiple loop fusion models with cost functions to maximize locality and parallelism, these models involve separate optimization steps rather than seamlessly integrating with other loop transformations like loop permutation, scaling, and shifting. Incorporating parallelism-preserving loop fusion heuristics into existing affine transformation frameworks like Pluto, LLVM-Polly, PPCG, and PoCC requires solving a large number of Integer Linear Programming formulations, which increase auto-transformation times significantly.

In this work, we incorporate polynomial time loop fusion heuristics into the Pluto-lp-dfp framework. We present a data structure called the fusion conflict graph (FCG), which enables us to efficiently model loop fusion in the presence of other affine loop transformations. We propose a clustering heuristic to group the vertices of the FCG, which further enables us to provide three different polynomial time greedy fusion heuristics, namely, maximal fusion, typed fusion, and hybrid fusion, while maintaining the compile time improvements of Pluto-lp-dfp over Pluto. Our experiments reveal that the hybrid fusion model, in conjunction with Pluto's cost function, finds efficient transformations that outperform PoCC and Pluto by mean factors of $1.8 \times$ and $1.07 \times$, respectively, with a maximum performance improvement of $14 \times$ over PoCC and $2.6 \times$ over Pluto.

CCS Concepts: • Software and its engineering $\rightarrow$ Compilers; Source code generation;

Additional Key Words and Phrases: Affine transformations, pluto algorithm, fusion conflict graph

ACM Reference format:

Aravind Acharya, Uday Bondhugula, and Albert Cohen. 2020. Effective Loop Fusion in Polyhedral Compilation Using Fusion Conflict Graphs. ACM Trans. Archit. Code Optim. 17, 4, Article 26 (September 2020), 26 pages.

https://doi.org/10.1145/3416510

\footnotetext{
Aravind Acharya was also affiliated with NVIDIA Corporation at the time of publication. Email: aravinda@nvidia.com. We acknowledge CEFIPRA (the Indo-French Centre for Promotion of Advanced Research) for a research grant and INRIA for its Associate Team award, funding the collaboration between the Indian Institute of Science and INRIA.

Authors' addresses: A. Acharya and U. Bondhugula, Department of Computer Science and Automation, Indian Institute of Science, Bengaluru, Karnataka 560012, India; emails: \{aravind, udayb\}@iisc.ac.in; A. Cohen, Google, 8 rue de Londres, Paris 75009, France; email: albertcohen@google.com.

Permission to make digital or hard copies of all or part of this work for personal or classroom use is granted without fee provided that copies are not made or distributed for profit or commercial advantage and that copies bear this notice and the full citation on the first page. Copyrights for components of this work owned by others than the author(s) must be honored. Abstracting with credit is permitted. To copy otherwise, or republish, to post on servers or to redistribute to lists, requires prior specific permission and/or a fee. Request permissions from permissions@acm.org.

(C) 2020 Copyright held by the owner/author(s). Publication rights licensed to ACM.

1544-3566/2020/09-ART26

https://doi.org/10.1145/3416510
}

ACM Transactions on Architecture and Code Optimization, Vol. 17, No. 4, Article 26. Publication date: September 2020. 


\section{INTRODUCTION}

Affine transformation algorithms have gained significant interest in recent times, in the areas of domain-specific languages and general-purpose compilation. Optimizing affine loop nests has been the target of many affine transformation frameworks. Recently, the MLIR [30] intermediate representation's design incorporated concepts from affine structures such as expressions, maps, and sets to make it easy to develop mid-level transformations and analyses-those that rely on restructuring loop nests and multi-dimensional arrays.

Modern multicore processors pose significant challenges for an optimizing compiler to efficiently utilize on-chip resources like multiple cores, SIMD units and deep memory hierarchies to extract maximal performance. Affine transformation frameworks incorporate various cost models $[5,11,12,21,23,24,43]$, to automatically find loop transformations that either maximize parallelism and locality in conjunction with other criteria. These also include the state-of-the-art algorithms implemented in auto-transformation frameworks like Pluto [6], Pluto+ [3], ISL and PPCG [44, 48], R-Stream [29, 43], and PoCC [20, 36]. However, these affine transformation frameworks lack the infrastructure to efficiently model loop fusion without significant compile-time overheads. However, older and traditional loop transformation approaches model loop fusion efficiently $[17,18,19,25,40]$, with the primary objective of maximizing locality and parallelism. However, these approaches are primarily restricted to perfect loop nests and consider a limited set of transformations in conjunction with fusion. Hence, a framework to efficiently model fusion of imperfectly nested loops in conjunction with transformations such as loop permutation, scaling, and shifting, without significant compile time overhead has been missing.

Our recently proposed Pluto-lp-dfp [1] framework addresses the scalability issue in the Pluto scheduling algorithm by decoupling the auto-transformation phase into three components: (1) loop fusion and permutation, (2) loop scaling and shifting, and (3) loop skewing. At each phase, the framework used a Linear Programming (LP) formulation instead of Integer Linear Programming (ILP). This decoupling in the Pluto-lp-dfp framework also comes with an added advantage that we leverage: it allows loop fusion to be modeled in conjunction with loop permutation, scaling and shifting. The time complexity of Pluto-lp-dfp is polynomial in the number of polyhedral statements, provided the loop fusion and permutations are found in polynomial time. In the rest of this section, any approach that is polynomial in the number of polyhedral statements is referred to as a polynomial time approach.

In this work, we introduce a data structure called the fusion conflict graph (FCG), which is central to our approach for finding loop permutations in polynomial time, while modeling loop fusion efficiently. The vertices in this graph represent dimensions of statements and the edges represent the dimensions that cannot be fused and permuted to the outermost level. We observe that nonadjacent vertices of the FCG, when grouped together in a specific way (described in Section 3), represent the set of dimensions that can be fused together and permuted to the outermost level. We incorporate SCC-based statement clustering heuristics to group the vertices of the FCG. This clustering not only reduces the number of vertices in the FCG but also provides a polynomial time approach to find loop permutations. We also incorporate greedy variants of a maximal fusion heuristic called max-fuse, a parallelism preserving fusion heuristic typed-fuse, and a hybrid fusion variant called hybrid-fuse, which is a combination of max-fuse and typed-fuse variants. We observe that in the case of time-iterated stencils, due to the presence of tile-wise concurrent start, parallelism-preserving fusion heuristics do not perform well due to loss of locality and absence of communication-free parallel loops. Hence, we provide a characterization to identify stencil dependence patterns in programs and use a different heuristic in such program segments. We compare the performance of our fusion models with the recent work of Kong et al. [20], which we refer to as 
PoCC + in this article, and an optimized version of Pluto with improved intra-tile optimizations and support for multi-loop unroll and jam optimization. The hybrid-fuse model outperforms PoCC+ and Pluto by a geomean factor of $1.8 \times$ and $1.07 \times$, respectively, on benchmarks from PolyBench and NAS benchmark suites, while maintaining significant compile time improvements of Plutolp-dfp over Pluto. In some cases, the transformations found by our fusion heuristic outperform Pluto by $2.6 \times$. For the NAS benchmarks, the hybrid-fuse model is faster than Pluto by a geomean factor of $234 \times$ over Pluto in terms of compilation time. In these benchmarks, PoCC + failed to find a transformation in a reasonable amount of time. The contributions of this article are as follows:

- We present the fusion conflict graph and its application to the embedding of traditional loop fusion models into the Pluto algorithm for automatically finding profitable affine loop transformations.

- We introduce a clustering heuristic to group the vertices of the FCG. Using the clustered FCG, we not only find permutations in polynomial time but also incorporate parallelismpreserving fusion heuristics to work in tandem with loop permutation, loop scaling, and loop shifting.

- We provide a characterization for stencils that have tile-wise concurrent start and apply a different fusion heuristic for program segments that contain these stencil patterns.

- Our fusion model, when implemented in the Pluto-lp-dfp framework, outperforms the current state-of-the-art without any significant compile time overhead.

The rest of this article is organized as follows: Section 2 provides the necessary background on polyhedral compilation and the Pluto-lp-dfp framework. Section 3 details the construction of the FCG and our approach to find loop permutations using greedy heuristics. In Section 4, we describe our implementation of typed fusion while taking affine transformations like permutation, scaling and shifting into account. Experiments are presented in Section 5, related work in Section 6, before conclusions in Section 7.

\section{BACKGROUND}

In this section, we provide the necessary background on the polyhedral model and the Pluto-lp-dfp framework.

\subsection{Polyhedral Model}

The polyhedral model has been widely used to optimize loop nests because of its ability to compose a variety of loop transformations. In the polyhedral model, every statement $S$ in a loop nest has an iteration domain, or an index set I. For example, consider the code snippet of the gemver kernel from PolyBench suite shown in Figure 1(a). The domains of statements $S_{1}$ and $S_{2}$, given by

$$
I_{S_{1}}=I_{S_{2}}=\{[i, j]: 0 \leq i, j \leq N-1\},
$$

define a two-dimensional iteration space where $i$ and $j$ are loop iterator variables and $N$ is a program parameter. An iteration vector $\overrightarrow{i_{S}}$ is used to represent a dynamic instance of the statement $S$. Each component of $\overrightarrow{i_{S}}$ corresponds to the loops surrounding the statement $S$ from outermost to innermost. Let $m_{S}$ denote the number of components in $\overrightarrow{i_{S}}$, which we refer to as the dimensionality of the statement $S$. Programs that we consider have Static Control Paths (SCoPs), i.e., loop bounds and array access functions are affine combinations of the outer loop iterator variables and program parameters. A loop around a statement $S$ corresponds to hyperplane in the iteration space of $S$. Two statements $S$ and $T$ are said to be data dependent if there are instances $\overrightarrow{i_{S}}$ and $\overrightarrow{i_{T}}$ such that, $\overrightarrow{i_{S}}$ and $\overrightarrow{i_{T}}$ access the same location and one of the accesses is a write. A Data Dependence Graph 


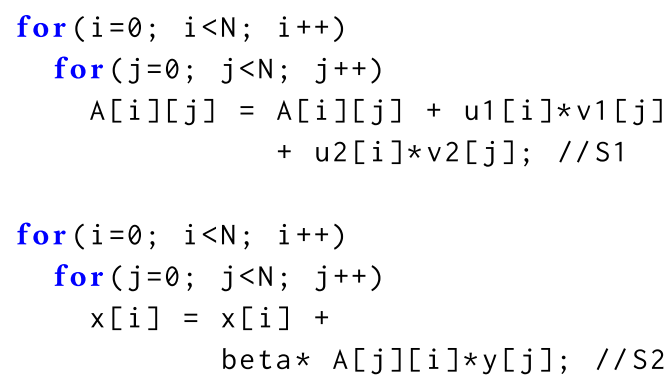

(a) Gemver code.

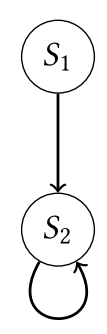

(b) DDG.
Valid permutation:

$T_{S_{1}}:(i, j) \rightarrow(i, j)$

$T_{S_{2}}:(i, j) \rightarrow(j, i)$

Invalid permutation:

$T_{S_{1}}:(i, j) \rightarrow(i, j)$

$T_{S_{2}}:(i, j) \rightarrow(i, j)$

Permutation with outer parallelism:

$$
\begin{aligned}
& T_{S_{1}}:(i, j) \rightarrow(j, i) \\
& T_{S_{2}}:(i, j) \rightarrow(i, j)
\end{aligned}
$$

(c) Permutations of the loop nest.

Fig. 1. Snippet from Gemver kernel.

(DDG), $G\left\langle G_{V}, G_{E}\right\rangle$, is a graph whose vertices are the set of statements in the program. A data dependence between two statements in the program corresponds to an edge in the DDG. For the gemver example, the DDG is shown in Figure 1(b). Data dependencies in an affine loop nest can be represented as data dependence relations, which are a conjunction of constraints. These constraints are affine combinations of loop iterator variables of the the source and target iterations, program parameters, which are symbols that do not change in the polyhedral part of the program being analyzed, and existentially quantified variables. Thus each edge $e$ in the DDG is associated with a set of constraints, also called the dependence polyhedron $D_{e}$. The union of all dependence polyhedra precisely represents the set of all data dependencies in the program.

\subsection{Affine Transformations}

Affine transformations can be used to model a rich class of loop reorderings with various objectives that either enhance locality and parallelism, minimize latency and synchronization or combinations of these. Formally, a multi-dimensional affine transformation for a statement $S$ in a program is an affine function of input loop iterators surrounding the statement $S$ and program parameters, which are represented in Figure 2.

Examples of various loop transformations are shown in Figure 3. Note that every transformation shown in Figure 3 can be obtained by choosing suitable integral values for $c_{i j}$ s, which we refer to as transformation coefficients, and the matrix of transformation coefficients is called the transformation matrix. Each row in this transformation matrix represents a hyperplane or a level of the transformation. Whenever we refer to the transformation at a given level, we omit the row identifier in the transformation coefficients. Polyhedral auto-transformation frameworks aim at finding these transformation coefficients. We use the notation $c_{S}^{i}$ to represent the transformation coefficient corresponding to dimension $i$ of the statement $S$.

\subsection{Pluto-Ip-dfp Framework}

The Pluto algorithm [6] iteratively finds hyperplanes, $\phi_{S}$, for each statement $S$, that satisfy the tiling validity condition:

$$
\phi_{S_{j}}(\vec{t})-\phi_{S_{i}}(\vec{s}) \geq 0,
$$

for every dependence $\langle\vec{s}, \vec{t}\rangle \in D_{S_{i} \rightarrow S_{j}}$. Intuitively, these tiling validity constraints ensure that the dependence vectors in the transformed space do not have any negative components, and hence, the loop nest could be tiled. Consecutive hyperplanes that satisfy the tiling validity constraints form a tileable band. There may exist many possible hyperplanes that satisfy the tiling validity condition. The Pluto algorithm finds hyperplanes that place dependent statement instances as close to each 
- Loop permutation

$$
T_{S}=\left(\begin{array}{ll}
c_{01} & c_{02} \\
c_{11} & c_{12}
\end{array}\right) \cdot\left(\begin{array}{l}
i \\
j
\end{array}\right)+\left(\begin{array}{l}
c_{00} \\
c_{10}
\end{array}\right)
$$

Fig. 2. Affine transformation for a two-dimensional statement with iterators $i$ and $j$. $(i, j) \rightarrow(j, i)$

- Loop skewing

$(i, j) \rightarrow(i, i+j)$
- Loop shifting

$(i, j) \rightarrow(i, j+1)$

- Loop scaling

$(i, j) \rightarrow(i, 2 * j)$

Fig. 3. Examples of affine transformations.

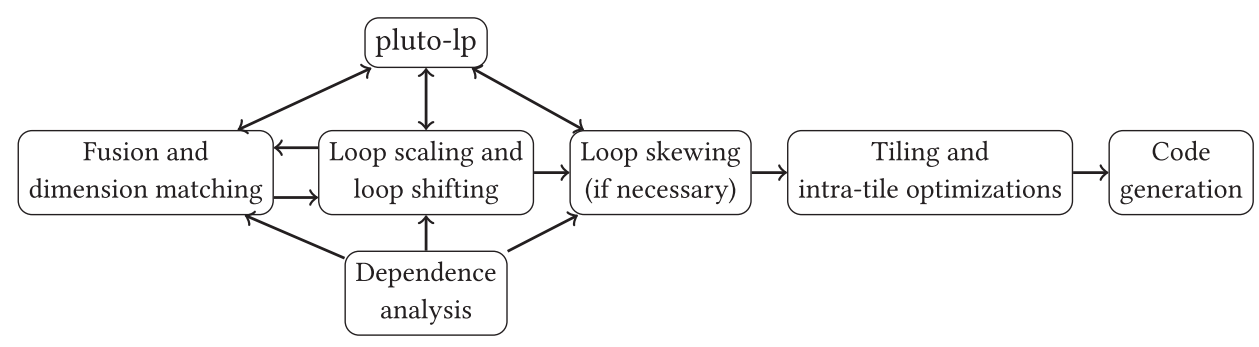

Fig. 4. Pluto-Ip-dfp framework.

other as possible. This is first achieved by defining an upper-bound on the dependence distance for every dependence as follows:

$$
\phi_{S_{j}}(\vec{t})-\phi_{S_{i}}(\vec{s}) \leq \vec{u} \cdot \vec{p}+w
$$

where $\vec{p}$ denotes the program parameters, and $\vec{u}$ and $w$ are variables in the ILP formulation. To minimize the dependence distance, the Pluto algorithm minimizes the coefficients $\vec{u}$ and $w$ by finding the lexicographic minimum (lexmin) of $(\vec{u}, w)$ as the objective function of the ILP formulation. The constraints in this ILP formulation include tiling validity constraints and constraints that enforce linear independence of transformation hyperplanes in the non-negative orthant. Note that these dependence distances are always non-negative and hence, the lower bounds of $\vec{u}$ and $w$ are $\overrightarrow{0}$ and 0 , respectively. If the hyperplane $\vec{h}$ at a level $\ell$ is such that $\vec{u}, w=(\overrightarrow{0}, 0)$, then the resulting hyperplane is outer parallel, because an iteration of the loop at level $\ell$ contains both the source and the target iterations of every dependence, resulting in a communication-free parallel loop; otherwise the dependence distance would be non-zero, and the solution of the ILP formulation would either have a non-zero value for $\vec{u}$ or $w$. Using the above ILP formulation, the Pluto algorithm finds hyperplanes level by level from outermost to innermost.

While the Pluto algorithm enforces linear independence of tiling hyperplanes, the Pluto-lp-dfp framework [1] decouples the auto-transformation framework into fusion and permutation, scaling and shifting, and loop skewing components as shown in Figure 4. The output of each phase is a transformation matrix. The Pluto-lp-dfp framework takes polyhedral dependencies as input and the output is a transformation for every statement in the program. The first step in Pluto-lp-dfp is to find a valid permutation. ${ }^{1}$ A permutation at a given level is valid if and only if there are loop scaling and shifting factors in the permutation such that the resulting transformation (with scaling and shifting factors) will not violate any dependencies. Formally, a permutation $\mathbb{P}$ for a statement $S$ is valid, if and only if for each level $\ell$, the hyperplane $\phi$ at $\ell$ satisfies the condition:

$$
\exists k_{1}, k_{2}, k_{3}, k_{4} \in \mathbb{N} .\left(k_{1} \times \phi_{S_{t}}(\vec{t})+k_{2}\right)-\left(k_{3} \times \phi_{S_{s}}(\vec{s})+k_{4}\right) \geq 0,
$$

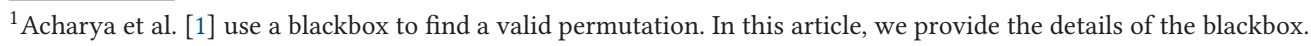


for every dependence $\langle\vec{s}, \vec{t}\rangle \in D_{S_{s} \rightarrow S_{t}}$. Here, $k_{1}$ and $k_{3}$ are called the loop scaling factors while $k_{2}$ and $k_{4}$ are called the loop shifting factors. The output of the first stage is a permutation matrix. For each level $\ell$, the scaling and shifting stage in the Pluto-lp-dfp framework finds loop scaling and shifting factors $\left(k_{1}, k_{2}, k_{3}\right.$, and $\left.k_{4}\right)$ for each level in the permutation using an LP formulation, which we refer to as pluto-lp. The constraints in this LP include tiling validity constraints, dependence distance bounding constraints (Equations (2) and (3)) and few others representing the permutation. The objective function of this LP is to find the lexmin of $(\vec{u}, w)$, which is the same as Pluto. The optimal solution of this LP formulation is guaranteed to be rational and this rational solution and be scaled to integral solution. Loop skewing is introduced by Pluto-lp-dfp if and only if it enables loop tiling. This is very important in time-iterated stencils, where loop skewing transformations not only enable tiling but also tile-wise concurrent start through diamond tiling [2]. Once the loop nest is tiled, intra-tile optimizations are performed to improve spatial reuse and vectorization. The time complexity of the Pluto-lp-dfp framework is polynomial in the sum of dimensionalities of polyhedral statements in the program, which we refer to as polynomial time in the rest of the article, provided valid permutations are found in polynomial time.

In the rest of this article, we describe an approach to find valid permutations, and more preferably the ones that maximize locality and parallelism. For example, consider the gemver kernel shown in Figure 1. The two statements are fully distributed and the $j$ loop of $S_{2}$ is sequential. The two loop nests can be completely fused by a combination of loop interchange and loop fusion transformations. This can be achieved using valid permutations shown in Figure 1(c). The first valid permutation completely fuses the statements after interchanging the $i$ and $j$ loops of $S_{2}$ and thus the write in statement $S_{1}$ will be available in the register for the read in statement $S_{2}$. The last permutation is similar to the first one, as it interchanges loops $i$ and $j$ of $S_{1}$ instead of $S_{2}$ yielding a fused, communication-free loop nest. The second permutation in Figure 1(c) fuses the loop $i$ of statements $S_{1}$ and $S_{2}$ at the outermost level. This permutation is incorrect, because, in the fused loop nest, the read of array $A$ in $S_{2}$ will happen before the write in $S_{1}$ violating the RAW dependence between $S_{1}$ and $S_{2}$; for example, in the iteration $(i=0, j=1)$, the loop nest corresponding to the invalid permutation would perform the read in $S_{2}$ but the corresponding write at statement $S_{1}$ would happen in the iteration $(i=1, j=0)$. Formally, the permutation at the outermost level is not valid, because there does not exist loop scaling and shifting factors that satisfy Equation (4). The goal of this article is to find profitable valid permutations in polynomial time.

\section{FINDING VALID PERMUTATION}

In this section, we describe our approach to find a valid permutation in the Pluto-lp-dfp framework. A permutation $\mathbb{P}$ is said to be valid if there are loop scaling and loop shifting factors for each level in $\mathbb{P}$, such that the resulting transformation after scaling and shifting, will not violate any dependencies. The objective of finding a good permutation is to enhance locality by enabling loop tiling, fusion and to improve parallelism. Since loop fusion/distribution decisions are also made at this stage, a framework that allows to model various fusion opportunities is desirable. With this objective, we present a data structure called the fusion conflict graph, which aids in efficiently modeling loop fusion, while looking for valid permutations.

\subsection{Fusion Conflict Graph}

In this section, we provide the formal definition of the fusion conflict graph and its properties that enable us to find valid permutations. A fusion conflict graph (FCG) is an undirected graph $F\langle V, E\rangle$, where the set of vertices is given by $V=\left\{S_{1}^{1}, S_{1}^{2}, \ldots, S_{1}^{m_{S_{1}}}, S_{2}^{1}, \ldots, S_{n}^{m_{S_{n}}}\right\}$, i.e, each vertex corresponds to a dimension of a statement in the program. An edge between $S_{s}^{i}$ and $S_{t}^{j}$ represents that the $i$ th dimension of $S_{s}$ and $j$ th dimension of $S_{t}$ cannot be fused together and permuted to the 


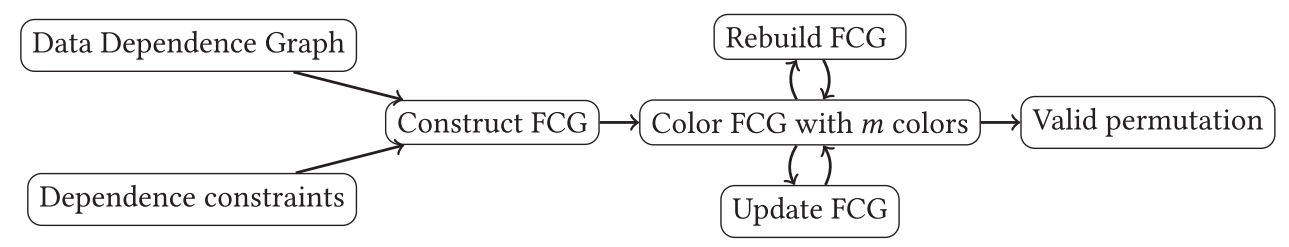

Fig. 5. Our approach to find a valid permutation.

outermost level. If the loop nest can be fully permuted, then it can be tiled as well. Hence, an edge in the fusion conflict graph encodes invalidity of loop fusion and tileability.

Once the FCG is constructed, the objective is to find convex independent sets of the FCG, which correspond to a valid permutation of the input program (cf. Theorem 3.2). Given an FCG, we say that an independent set $\mathcal{I}$ of the FCG is convex if for each $S_{t}^{i} \in \mathcal{I}$, the following condition holds:

$$
\forall S_{s} .\left(S_{s}, S_{t}\right) \in G_{E}, \exists S_{s}^{j} \in \mathcal{I},
$$

where $G_{E}$ is an edge in the DDG. Intuitively, if a vertex of the FCG corresponding to a dimension $i$ of a statement $S_{t}$ is present in $\mathcal{I}$, then, for every predecessor $S_{s}$ of $S_{t}$ in the DDG, there must exist some vertex $S_{s}^{j}$ in $\mathcal{I}$. Each convex independent set represents the set of vertices that can be fused and permuted to the outermost level. Any pair of non-adjacent vertices $S_{s}^{i}, S_{t}^{j} \in \mathcal{I}$, indicate that fusing these dimensions will not violate any dependence between $S_{s}$ and $S_{t}$. Convexity of $\mathcal{I}$ ensures that transitivity of dependencies is not violated. We obtain a convex independent set by a convex coloring of the FCG. Given a fusion conflict graph, we say that the coloring of the fusion conflict graph is convex, if the vertices with the same color form a convex independent set. In the rest of the article, we refer to convex coloring of the FCG as coloring of the FCG. Note that the goal of coloring is to obtain convex independent sets, which in turn corresponds to a valid permutation, which is the output of the first phase of the Pluto-lp-dfp framework.

3.1.1 Overview. The overview of our approach is shown in Figure 5. The first step is to construct the fusion conflict graph using the dependence graph and dependence constraints (described in Section 3.2). We use the term dependence constraints to represent union of tiling validity constraints and dependence distance bounding constraints given in Equations (2) and (3).

Consider the fdtd-2d benchmark from the PolyBench benchmark suite shown in Figure 6 and the corresponding DDG is shown in Figure 6(b). Figures 6(c) and 6(d) show two possible valid permutations. The first one involves loop distribution at the second level, ${ }^{2}$ while the second one not only fuses all the statements but also enables loop skewing and diamond tiling in the later stages of the Pluto-lp-dfp framework. These valid permutations can be obtained using a convex coloring of the FCG shown in Figure 7(a). The coloring is performed level by level and is guided by the topological order of the SCCs to enforce convexity of the coloring routine. The upper bound on the number of colors to be used for coloring is given by $m$, where $m$ is the maximum of dimensionalities of all statements in the program. This establishes a mapping of colors to dimensions of the loop nest. We assume that the colors are ordered; ordering of colors gives the permutation for every statement from the outermost level to the innermost. If the loop nest can be completely fused and tiled, then FCG can be successfully colored with $m$ colors. The vertices that obtain the same color represent the dimensions that can be fused and permuted to the outermost level. FCG may not be colorable with $m$ colors, either due to a permute preventing or a fusion preventing dependence. If coloring

\footnotetext{
${ }^{2}$ Loop distribution at a level $\ell$ is modeled using scalar hyperplanes, which corresponds to a transformation with all 0 's except for the coefficient $c_{0}$ of statement $S$. The statements in the transformed program are arranged in the increasing order of $c_{0} \mathrm{~s}$.
} 


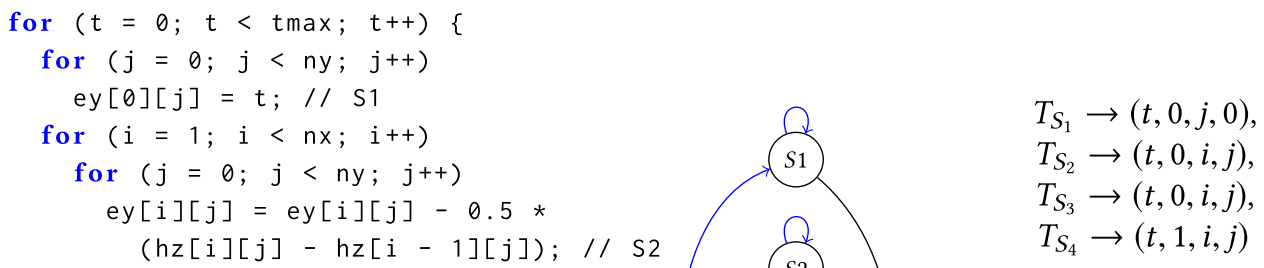

$$
\begin{aligned}
& T_{S_{1}} \rightarrow(t, 0, j, 0), \\
& T_{S_{2}} \rightarrow(t, 0, i, j), \\
& T_{S_{3}} \rightarrow(t, 0, i, j), \\
& T_{S_{4}} \rightarrow(t, 1, i, j)
\end{aligned}
$$

(a) Fdtd-2d benchmark.

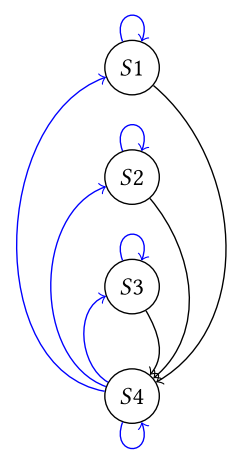

(b) DDG. (c) Permutation with loop distribution.

$$
\begin{aligned}
T_{S_{1}}(t, j) & \rightarrow(t, j), \\
T_{S_{2}}(t, i, j) & \rightarrow(t, j, i), \\
T_{S_{3}}(t, i, j) & \rightarrow(t, j, i), \\
T_{S_{4}}(t, i, j) & \rightarrow(t, j, i)
\end{aligned}
$$

(d) Permutation enabling diamond tiling.

Fig. 6. FCG construction and coloring in fdtd-2d benchmark.

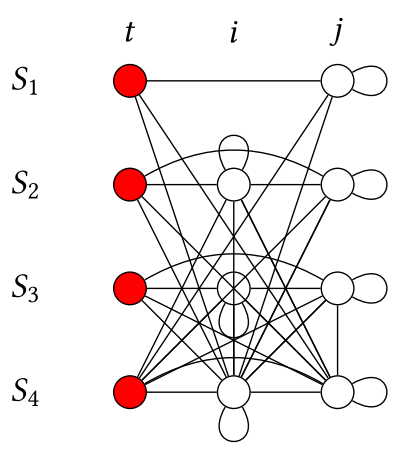

(a) FCG constructed by Algorithm 1.

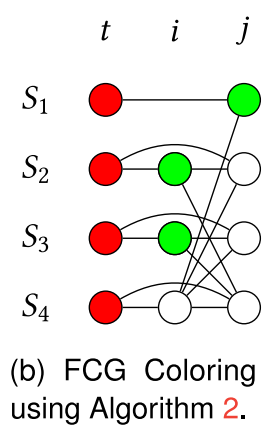

using Algorithm 2.

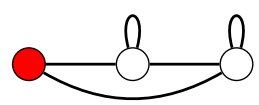

(c) FCG with the clustered approach.

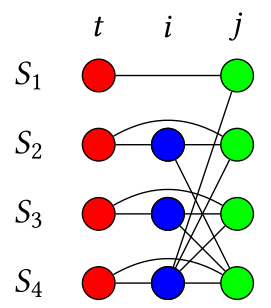

(d) Greedy coloring in fdtd-2d.

Fig. 7. FCGs of the fdtd-2d benchmark during various stages of the Pluto-Ip-dfp framework.

with a color $c$ failed due to a permute preventing dependence, then dependencies that are satisfied at outer levels are removed and the FCG is rebuilt. Now, the dimension corresponding to vertices that are colored with $c$ can be permuted only with the loops at lower levels. Intuitively, rebuilding the FCG marks the beginning of a permutable band of loops. If coloring failed due to a fusion preventing dependence, then loops are distributed and the DDG and the FCG are updated. Once all the vertices are colored, the ordering of colors can be used to obtain the permutation. First in Section 3.3, we describe how the permutation shown in Figure 6(c) can be obtained by a simple coloring routine. Then later in Section 3.4.1 we describe the details of the greedy coloring routine, which helps to obtain the permutation shown in Figure 6(d). All design choices described in the rest of this section are aimed at maximizing fusion without loss of parallelism using polynomial time routines.

\subsection{Construction of the Fusion Conflict Graph}

In this section, we describe the construction of the fusion conflict graph. Recall that, each dimension of a statement in the program corresponds to a vertex in the FCG. An edge in the FCG 
represents the dimensions that cannot be fused and permuted to the outermost level. Algorithm 1 incrementally constructs the fusion conflict graph by adding edges between vertices of the FCG in multiple stages. The first stage adds intra-statement permute preventing edges and the second stage adds inter-statement permute and fusion preventing edges.

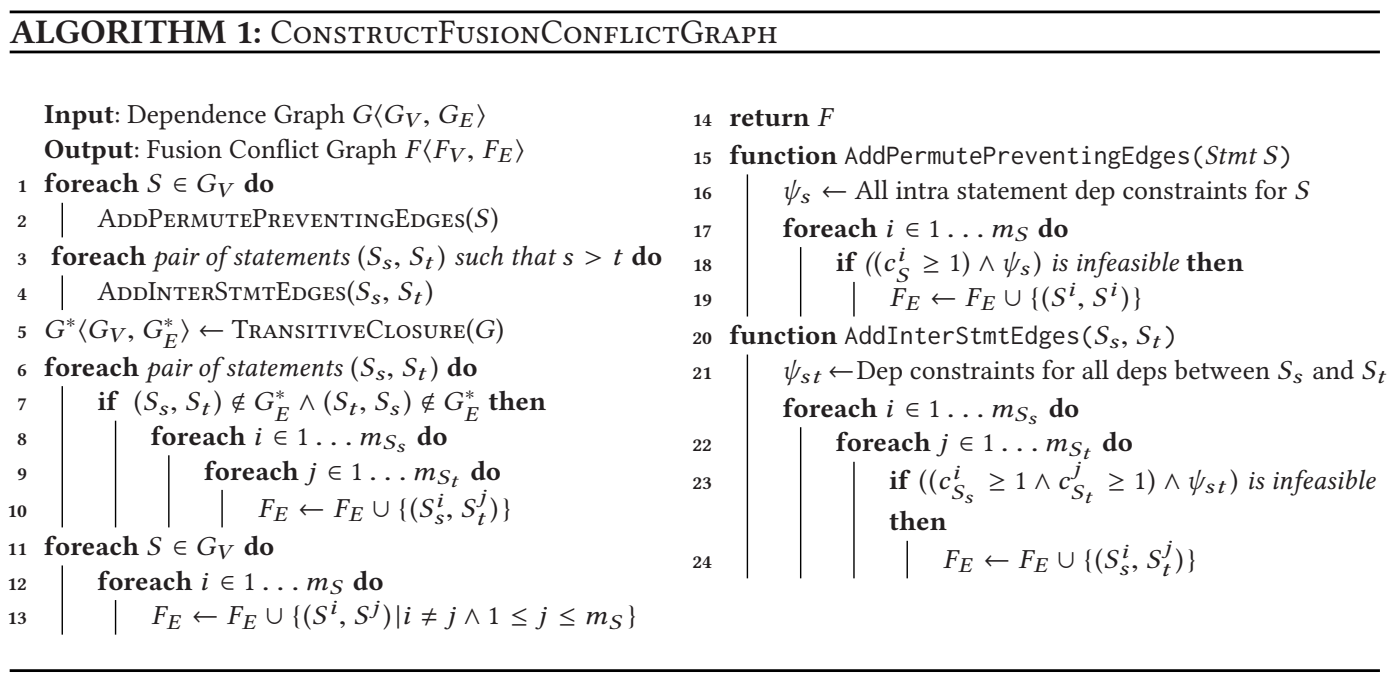

Adding intra-statement edges: Algorithm 1 adds intra-statement permute preventing edges in the routine AddPermutePreventingEdges for each dimension of a statement $S$. Using the DDG $G$, the routine collects all intra-statement dependencies for $S$ as shown in Equation (6):

$$
D_{S} \equiv\left\{D_{e} \mid e=(S, S) \in G_{E}\right\},
$$

where $e$ is a self edge on a statement $S$ in $G$. For each dependence in $D_{S}$, dependence constraints are constructed, which include both tiling validity constraints and dependence distance bounding constraints and is denoted by $\psi_{s}$ in Algorithm 1 (line 16). If a dimension $i$ is not permutable to the outermost level, then it has a negative component along $i$ for at least one intra-statement dependence. To find if $i$ is permutable to the outermost level, we set the lower bound of the coefficient $c_{S}^{i}$, to 1 . Coefficients corresponding to other dimensions of $S$, except $c_{S}^{0}$ (loop shifting coefficient), are set to zero. Note that, none of the transformation coefficients are constrained to be integers. The satisfiability of these constraints is checked using an LP formulation with the same objective as Pluto. As described by Acharya et al. [1], the existence of a rational solution implies that there exists an integer solution and vice versa. Therefore, if these constraints are unsatisfiable, then dimension $i$ is not permutable. Hence, we add a self edge on the vertex $S^{i}$ in the FCG (line 18), which prevents coloring of the vertex $i$ in the coloring phase. This edge will be removed only when permute preventing dependencies are satisfied at some outer level. Note that if we are considering just feasibility of constraints, then dependence distance bounding constraints can be removed in the above formulation. However, we intend to solve these constraints as an LP with the same objective as Pluto, because it allows us to model parallelism preserving loop fusion heuristics alongside loop permutations, loop scaling and loop shifting transformations, which we describe in Section 4. The objective function of the Pluto algorithm is meaningful only when dependence distances are upper bounded, and therefore, dependence distance bounding constraints are essential. In the rest of this article, whenever we check the satisfiability of a set of constraints, we solve the constraints using an LP, with the same objective function as the Pluto algorithm. In the case of the fdtd-2d benchmark the FCG for which is shown in Figure 7(a), the permute preventing edge on the vertex 
$S_{1}^{j}$ indicates that the dimension $j$ of $S_{1}$ cannot be permuted to the outermost level, as it violates the WAW dependence on $S_{1}$ carried by the outermost $t$ loop. Similarly, permute preventing edges are also added on vertices corresponding to dimensions $i$ and $j$ of statements $S_{2}, S_{3}$ and $S_{4}$.

Adding inter-statement edges: Algorithm 1 adds inter-statement permute and fusion preventing edges in the routine AddinterStmtEdges. For each pair of statements $S_{s}$ and $S_{t}$ that are adjacent in the DDG $G$, it collects all dependencies (both intra and inter-statement dependencies) between them according to Equation (7):

$$
D_{s t} \equiv\left\{D_{e} \mid e=\left(S_{s}, S_{t}\right) \in G_{E} \vee e=\left(S_{t}, S_{s}\right) \in G_{E}\right\} .
$$

Validity of fusing dimension $i$ of $S_{s}$ with dimension $j$ of $S_{t}$ and permuting to the outermost level is checked by solving dependence constraints $\psi_{s t}$ along with the constraints $c_{s}^{i} \geq 1$ and $c_{t}^{j} \geq 1$ (line 23). Coefficients corresponding to other dimensions of statements $S_{s}$ and $S_{t}$ are set to zero. If the above constraints are unsatisfiable, then an edge is added between $S_{s}^{i}$ and $S_{t}^{j}$ in the FCG. In the case of the fdtd-2d example, the edge between $S_{1}^{j}$ and $S_{4}^{i}$ is added, because it is invalid to fuse the loop $j$ of $S_{1}$ with loop $i$ of $S_{4}$.

Fusing two statements that do not have any reuse tend to pollute caches, resulting in increase of conflict and capacity misses, increased register pressure and so on. Therefore, for any pair of vertices $\left(S_{s}, S_{t}\right)$, if there is no path in the DDG from $S_{s}$ to $S_{t}$ or vice versa, then fusion preventing edges are added between each and every dimension of $S_{s}$ and $S_{t}$ in the FCG (lines 6-10). These edges are added only when the FCG is constructed for the first time. Finally, the algorithm adds edges between vertices of the FCG that correspond to dimensions the same statement (line 13), ensuring that two dimensions of the same statement are not given the same color.

\subsection{Coloring the Fusion Conflict Graph}

In this section, we provide details of our convex coloring algorithm to obtain convex independent sets of the fusion conflict graph. We assume that SCCs in the DDG are numbered according to a topological ordering of SCCs. Vertices of the FCG are colored by the topological ordering of the SCCs to which the statements belong to enforce the convexity of the independent set obtained via coloring. We also assume that the colors are ordered, with smaller numbered colors representing outer dimensions.

Algorithm 2 colors the vertices of the FCG, one color at a time starting from the first SCC in the topological order. The routine ColorSCC (line 5) tries to color the vertices of the FCG corresponding to the statements SCC $i$ in the DDG. It returns true if the coloring succeeds for the SCC $i$; else it returns false. Note that the coloring routine that we describe in this section is dependent only on the input SCC $i$. Later, in Section 3.4.1, we describe a greedy coloring heuristic that takes into account the successor SCCs as well. Even for a single SCC, there may exist many dimensions that can be colored. The routine ColORSCC colors the outermost dimension in the original program order. At the outermost level, the coloring of the first SCC will succeed. This is because, for any given SCC $i$, there exists at least one dimension that fuses every statement in SCC $i$ without violating any dependencies. Consider the FCG from the fdtd-2d example shown in Figure 7(a). The coloring routine will color vertices corresponding to the dimension $t$ at the outermost level for all statements. Now, Algorithm 2 has colored vertices in the FCG from every statement in all SCCs in DDG with the color $c=1$. This corresponds to a valid permutation at the level $l=1$, which we prove formally in Section 3.5, and hence we update the transformation for each statement at the current level by setting $c_{S}^{i}$ to 1 , if the vertex $S^{i}$ in the FCG is colored with color $c$ (line 14). Algorithm 2 colors the FCG with the color next color $c+1$. At the second level, the coloring will fail because of the self edge on the vertex $S_{1}^{j}$. The routine IsPermutePreventing 


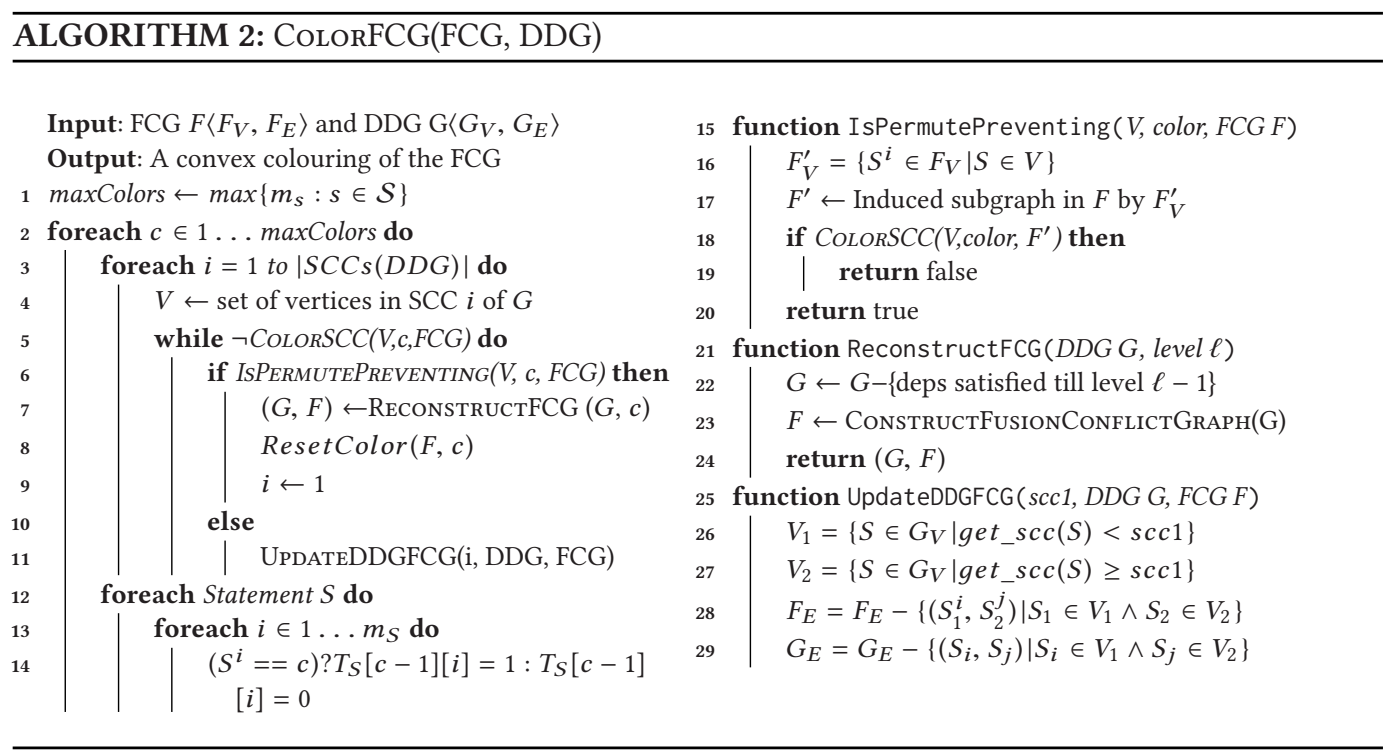

checks if vertices of the FCG corresponding to dimensions of statements in SCC $i$ can be colored in isolation. In the case of the fdtd-2d example, IsPermutePreventing also fails to color this SCC and hence returns true. Algorithm 2 removes those dependencies that are satisfied at the outer level $t$ and reconstructs the FCG (lines 22 and 23). The reconstructed FCG is shown in Figure 7(b). The reconstructed FCG now has four SCCs, each with a single statement. The colors of the vertices that are colored with $c$ are reset and the coloring is restarted from the first SCC (lines 8 and 9). The routine now colors the vertex $S_{1}^{j}$ with color 2, and then while coloring the vertices of the FCG from statement $S_{2}$, it has two choices; either $S_{2}^{i}$ or $S_{2}^{j}$. The routine ColorSCC colors vertex $S_{2}^{i}$ as it is the outermost dimension in the original program order that can be colored with the second color. Similarly, the vertex $S_{3}^{i}$ is colored as well. Now, neither $S_{4}^{i}$ nor $S_{4}^{j}$ can be colored and the routine ColorSCC fails to color the last SCC. The routine IsPermutePreventing returns false, because either of the vertices $S_{4}^{i}$ or $S_{4}^{j}$ can be colored in isolation. Therefore, the loop nests are distributed at this level, which is represented by the scalar value in the transformation shown in Figure 6(c). After distribution, the DDG is updated by removing the edges corresponding to the dependencies that are satisfied by this distribution and the FCG is reconstructed (lines 26-29). Now the routine COLORSCC successfully colors $S_{4}^{i}$ with the second color. The transformation matrix is updated for the level $\ell=2$. The vertices $S_{2}^{j}, S_{3}^{j}$ and $S_{4}^{j}$ are colored with the third color in the next iteration. The final transformation is shown in Figure 6(c).

The routine COLORSCC in Line 5 can fail for a maximum of two times for any given SCC; once due to a permute preventing edge and once due to a fusion preventing edge. Thus, the loop in Line 5 is guaranteed to terminate. The coloring routine described in Algorithm 2 is exponential in the size of the SCCs in the DDG. Moreover, if there exist many possible valid colorings for an SCC, then selecting one of these valid colorings becomes tedious. Suppose the goal is to locally fuse as many successors as possible for a given SCC $S$, then in the worst case, one would have to enumerate all possible colorings for every successor of $S$ and then decide the dimension of $S$ to be colored. In the case of the fdtd-2d benchmark, such a heuristic would also enable diamond tiling in a later stage of the Pluto-lp-dfp framework. Therefore, in the next section, we provide a clustering 


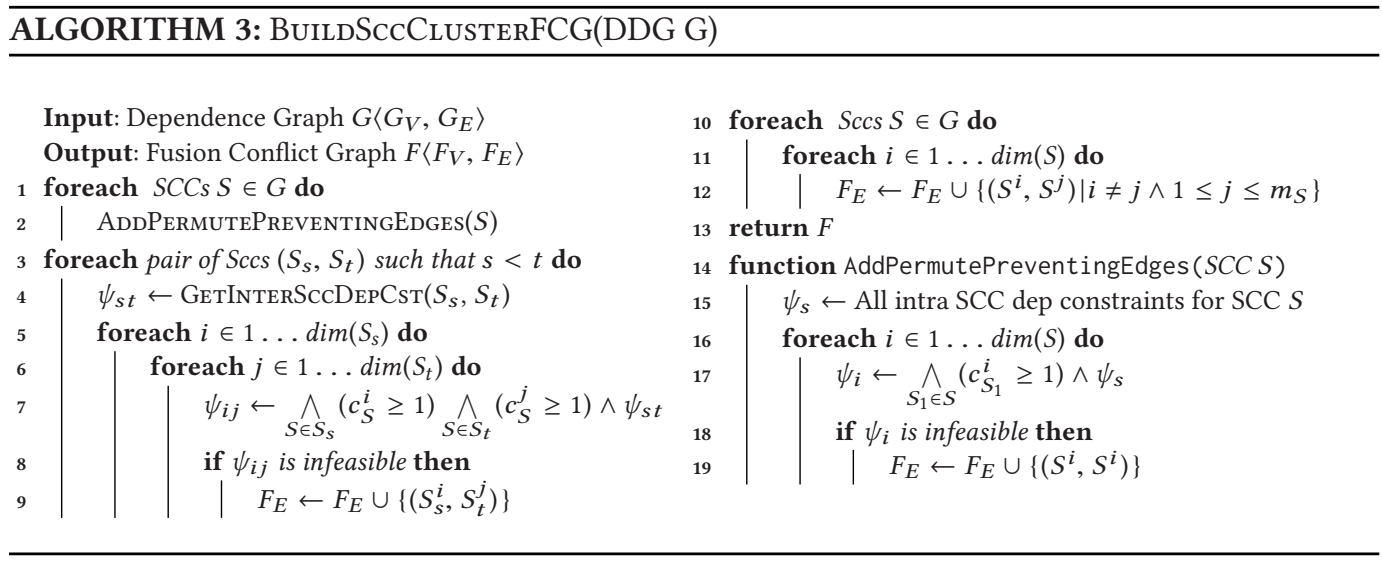

heuristic that not only results in a polynomial time SCC coloring routine but also makes way for simplistic implementation of greedy coloring heuristics.

\subsection{Clustering}

In this section, we describe a clustering heuristic to cluster the vertices of the FCG based on SCCs in the DDG. We then present a polynomial time greedy coloring heuristic, which can be efficiently implemented using the clustered FCG. Note that we use a different clustering heuristic than the one proposed by Mehta et al. [27], because their heuristic cannot model loop distribution efficiently, and hence can miss opportunities where loop distribution enhances parallelism. Later, in Section 4, we show how a parallelism-preserving fusion heuristic can be seamlessly incorporated in the clustered FCG, without affecting the coloring routine.

Our clustering heuristic is based on the observation that, for every statement in the SCC, there exists at least one dimension $i$ that fuses all the statements of the SCC without violating the tiling validity constraints. Therefore, if we assume that every statement in an SCC gets the same transformation at a given level, then all statements within an SCC can be clustered. We define dimensionality of an SCC to be the maximum of dimensionalities of all statements in the SCC. Each vertex in the clustered FCG corresponds to a dimension of an SCC in the DDG. Algorithm 3 depicts the construction of SCC clustered FCG. It is similar to Algorithm 1 but with a few minor changes. Addition of permute preventing edges is performed on a per-SCC basis by considering the intra-SCC dependencies, that is, the set of dependencies whose source and destination statements are within the given SCC. Note that all the statements in the SCC are assumed to have the same transformation, and hence, the lower bound of $c_{i}$ 's of all statements in the SCC is set to one (line 17). Inter-SCC fusion and permute preventing edges in the FCG are added for every pair SCCs $\left(S_{s}, S_{t}\right)$ in the DDG, by considering both intra and inter-SCC dependencies (lines 3-9). These edges are added for every pair of dimensions $(i, j)$ of SCCs $\left(S_{s}, S_{t}\right)$. Lower bounds of transformation coefficients corresponding to $i$ and $j$ of all statements in SCCs $S_{s}$ and $S_{t}$ are set to 1 . Transformation coefficients corresponding to all other dimensions, apart from the shifting coefficient, are constrained to 0 . Satisfiability of these constraints are checked with an LP formulation, in the same way as Algorithm 1, and edges in the FCG are added if the constraints are unsatisfiable. Algorithm 3 also adds edges in the FCG between vertices corresponding to SCCs that are not connected in the DDG (not shown in pseudocode). It also adds edges between vertices corresponding to dimensions of the same SCC (line 12). For example, in the case of the fdtd-2d example (cf. Figure 6(a)), at the outermost level, there is only one SCC and the corresponding FCG is shown 
in Figure 7(c). Intra-SCC permute preventing edges are added on vertices $S^{i}$ (and $S^{j}$ ) by setting the lower bounds of $c_{i} \mathrm{~s}$ (and $c_{j} \mathrm{~s}$ ) of all statements to 1 . We will present a greedy coloring heuristic in the following section to color the clustered FCG.

3.4.1 Greedy Coloring with Clustering in the Routine ColorSCC. Coloring of the clustered FCG is very similar to as in Algorithm 2. The obvious difference in the semantics of the coloring routine is that, when a vertex of the clustered FCG is colored, it defines a permutation for every statement in the SCC, instead of a single statement. Now the routine CoLORSCC will have $m$ choices to color a vertex and this coloring will enforce the permutation for all statements within the SCC. Apart from yielding a polynomial time coloring heuristic, clustering also has the following advantages: (1) simplification of permutability check in Line 6 of Algorithm 2, and (2) greedy coloring heuristics can be employed in a simplistic manner. We provide details on these advantages with an example.

The FCG for the fdtd-2d kernel constructed by Algorithm 3 is shown in Figure 7(c). Algorithm 2 colors the vertex corresponding to the time dimension $t$ in the first iteration, which is the only vertex that can be colored. Therefore, the permutation at the outermost level corresponds to the time dimension for each statement. Coloring the FCG with the second color fails due to permute preventing dependencies. This is inferred by the presence of self-edges on all the uncolored vertices of the FCG, namely, $S^{i}$ and $S^{j}$, in Figure 7(c). This check is very simple when compared to the check implemented by the routine IsPermutePreventing in Algorithm 2. The presence of permute preventing edges leads to removal of dependencies that are satisfied at the outermost level (shown in blue in Figure 6(b)). The updated DDG now has a single statement in every SCC. The reconstructed FCG is shown in Figure 7(d). Algorithm colors the only remaining dimension in SCC 1, and then, while coloring a vertex of SCC 2, it has two choices. Coloring vertex $S_{2}^{i}$ with the second color would enable us to color $S_{3}^{i}$ only, whereas coloring $S_{2}^{j}$ would enable coloring both $S_{3}^{j}$ and $S_{4}^{j}$. Here, the routine ColorSCC chooses the vertex that enables coloring of maximum number of successor SCCs in the topological order, which, in this example, is the vertex $S_{2}^{j}$. This greedy coloring heuristic is polynomial in the dimensionality and the number of SCCs in the DDG, only when clustering is incorporated. Such a greedy heuristic in the unclustered approach would be exponential, because coloring of vertices corresponding to statements within the given SCC as well as its successors must be considered. Thus, the main difference between the routine CoLORSCC described in this section and the one is Algorithm 2 is as follows: the greedy variant of the ColorSCC routine described in this section makes a coloring choice not only by considering the current SCC $S$ but also considers vertices of the FCG corresponding to the convex successors of $S$. Thus, the routine colors the vertex $S_{2}^{j}$ with the second color and proceeds by coloring the vertices $S_{3}^{j}$ and $S_{4}^{j}$. Then, in the last iteration, the algorithm colors the vertices $S_{i}^{2}, S_{3}^{i}$, and $S_{4}^{i}$. The final coloring obtained is shown in Figure 7(d), which corresponds to the permutation shown in Figure 6(d). This permutation leads to loop skewing and enables diamond tiling in a later stage of the Pluto-lp-dfp framework.

\subsection{Correctness}

In this section, we describe the correctness of our approach to find valid permutations using clustered FCG. Proofs that we provide here can also be extended to the unclustered approach. However, we exclude this from the article, as results presented in Section 5 are from the clustered approach only.

In this section, we first prove that for a given SCC, there exists at least one dimension that fuses all the statements of the SCC, without violating any dependencies. This is the assumption of our clustering heuristic. 
Theorem 3.1. Given a DDG $G$ of a program $P$, for each SCC $S$ in $G$, there exists at least one dimension $i$ that fuses all statements in $S$, without violating any dependencies.

Proof. We prove Theorem 3.1 by contradiction. Let us assume that there does not exist any dimension $i$ that fuses all statements belonging to SCC $S$, without violating any dependencies. However, in the input program, since the vertices are part of an SCC, there must exist a loop $i$, which carries a dependence along the back edge. This loop $i$ fuses all statements in $S$. Since the input program is correct, the dimension $i$ will not violate any dependence, and therefore, will satisfy the dependence constraints for all dependencies in the SCC.

The consequence of Theorem 3.1 is that, for every SCC $S$ in the DDG, there exists a vertex $v$ corresponding to some dimension $i$ of $S$ in the FCG without a self edge, at the outermost level. If there is a single SCC in the program, then $v$ can be colored with the color $c$ and this corresponds to a valid permutation at a particular level. The greedy coloring heuristic described in Section 3.4.1 is driven by a topological ordering of SCCs in the DDG, and therefore, the set of vertices that have the same color form a convex independent set. In Theorem 3.2, we prove that any convex independent set of the FCG found by the greedy coloring algorithm, corresponds to a valid permutation for every statement in the input program.

TheOrem 3.2. Every convex independent set $\mathcal{I}$ of the FCG $F$, corresponds to a valid permutation for the set of statements in the program $P$.

Proof. Let $\mathcal{S}=\left\{S_{1}, \ldots, S_{N}\right\}$ be the SCCs in the DDG whose corresponding vertices are present in $\mathcal{I}$, i.e, $\mathcal{S}=\left\{S \mid \exists j .1 \leq j \leq \operatorname{dim}(S) \wedge S^{j} \in \mathcal{I}\right\}$. Without loss of generality, let us assume that the dimension $i$ of every SCC in $\mathcal{S}$ is present in $\mathcal{I}$. Let $c$ be the color used for coloring at a level $\ell$. We will prove that, at any point of time in the coloring routine, whenever a vertex $S_{m}^{i}$ of the FCG is colored with $c$ (analogous to adding $S_{m}^{i}$ to $\mathcal{I}$ ), the set of vertices that are already colored with $c$ (analogous to elements in $\mathcal{I}$ ) represents a valid permutation for statements in those SCCs whose vertices corresponding to dimension $i$ have been colored. We will assume that it is valid to color $S_{m}^{i}$ with $c$ and the routine ColorSCC has succeeded. If $S_{m}^{i}$ was the first vertex added to $\mathcal{I}$, then by Theorem 3.1, it is a valid permutation. Let $S_{m}^{i}$ be the $m^{\text {th }}$ vertex added to $\mathcal{I}$. Let $k_{j}$ be the loop shifting factor required to shift and fuse dimension $i$ of SCCs $S_{j}$ and $S_{j-1}$, where $j<m$. Let $k$ be the maximum shifting factor, which is required to fuse SCC $S_{m}$ with its predecessors in the DDG. We make an observation that if two SCCs are connected in the DDG, then the target SCC can be delayed further by a loop shifting transformation without violating any dependencies. Therefore, when $S_{m}^{i}$ is colored with $c$, there exists an integer $k^{\prime}=k_{2}+k_{3}+\cdots+k_{m-1}+k$, that will be a valid loop shifting factor for every statement in $S_{m}$. Hence, there exists a loop shifting factor for every statement in every SCC of $\mathcal{S}$ such that dependencies are not violated. The existence of loop scaling factors can also be proved in a similar way. Once all the SCCs are colored, $\mathcal{I}$ corresponds to a valid permutation for every statement in the program.

Theorem 3.2 proves that a convex independent set of the FCG obtained via convex coloring represents a valid permutation, which is the output of the first phase of the Pluto-lp-dfp framework.

\section{TYPED FUSION}

The approach described in Section 3.4 constructs the FCG and the coloring heuristic greedily fuses as many statements as possible, under a given loop. However, this fusion strategy is not desirable, because it may inhibit parallelism. In this section, we provide a variant of typed fusion developed by Kennedy and McKinley [19], which ensures that loop fusion does not result in loss of parallelism. Our typed fusion heuristic allows fusion of a subset of loops that preserve parallelism 


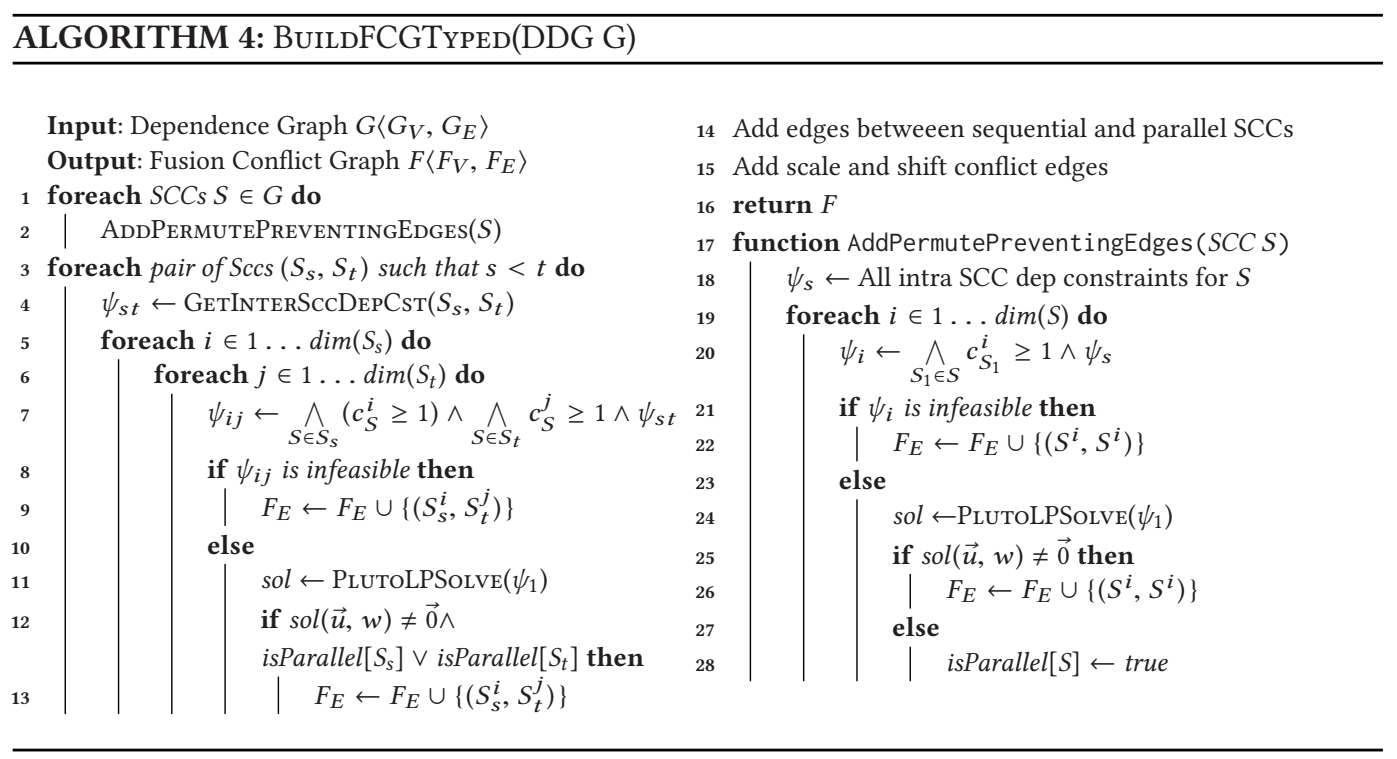

when compared to the approach described in Section 3.4, and hence, our approach adds parallelism preventing edges in the FCG, in addition to permute and fusion preventing edges.

\subsection{FCG Construction and Coloring}

Algorithm 4 provides details on construction of the FCG, which is very similar to Algorithm 3. The routine AddPermutePreventingEdges, in addition to adding permute preventing edges, adds parallelism preventing edges on vertices that correspond to serial dimensions (line 26). For a given SCC $S$, the routine AddPermutePreveningEdges, solves the same set of constraints as Algorithm 3, as a Linear Program (LP) with the same objective as Pluto (line 24), which is to find lexmin $\vec{u}, w$. If a non-zero solution for $\vec{u}$ or $w$ is obtained, then a parallelism preventing self-edge is added on the vertex $S^{i}$ in the FCG. In addition to adding parallelism preventing edges, the routine also marks an SCC as parallel if there is at least one parallel dimension along the canonical axes (Line 28). Once parallel SCCs are marked, parallelism preventing edges are also added if fusing two dimensions of two adjacent SCCs in the DDG do not result in a parallel loop (line 13). Parallelism preventing edges are also added between vertices of the FCG that correspond to dimensions of sequential and parallel SCCs (line 14). Analogous to Algorithm 3, Algorithm 4 also adds edges between SCCs that are not connected and between vertices corresponding to the dimensions of the same SCC (excluded in the pseudocode for the purpose of brevity).

Parallelism can also be lost by fusing SCCs that require different loop scaling or shifting factors. For example, consider the code shown in Figure 8(a). Statements $S_{1}$ and $S_{2}$ can be fused together by shifting $S_{1}$ by 1 with respect to loop $i$, while preserving parallelism, and similarly $S_{2}$ and $S_{3}$ can be fused with by shifting $S_{2}$ by 1 with respect to $i$. However, if all three statements are fused, then the resulting fused loop nest will not be parallel. This is because $S_{2}$ should be delayed from $S_{3}$ by 1 and $S_{1}$ should be delayed by 1 with respect to $S_{2}$ thereby resulting a net delay of 2 for the statement $S_{1}$ with respect to $S_{3}$. However, $S_{1}$ can be fused with $S_{3}$ only with a delay of 1 , while preserving parallelism. To account for such conflicting shifts, we add shift conflict edges in the FCG (Line 15 in Algorithm 4). This routine traverses SCCs in the reverse topological order, while summing up the shifts. The shifts for a pair of statements are computed during the addition of inter-SCC edges 
using the solution of the LP formulation (Line 11). Whenever the routine detects a conflicting shift, i.e two different shifting factors for fusing two dimensions of any two statements, it adds a parallelism preventing edge between dimensions of SCCs that requires a larger shift. In the above example parallelism preventing edge is added between $S_{1}^{i}$ and $S_{3}^{i}$ (dashed edge in Figure 8(b)). A similar approach is followed to add scale conflict edges in the FCG.

Once the FCG is constructed, we use the greedy coloring heuristic described in Section 3.4.1 to find convex independent sets. In the case of typed fusion, the coloring heuristic also ensures that if there exists a parallel hyperplane along the canonical axes, it is found at the outermost level, resulting in a communication-free loop nest. For the gemver kernel in Figure 1(a), with the typed fuse heuristic, the permutation with an outer parallel loop shown in Figure 1(c) is obtained. On a side note, the FCG can also be also used to model spatial locality by adding edges on vertices of the FCG that correspond to dimensions that exhibit spatial reuse. These edges can be removed in the coloring step, if the color corresponds to the innermost level. However, we perform intra-tile optimizations (cf. Section 4.4) to exploit spatial reuse.

\subsection{Stencil Characterization}

The typed fuse heuristic described in Section 4 distributes loop nests when fusion leads to loss of parallelism. However, there are scenarios where this distribution might result in loss of performance. For example, consider the jacobi-2d benchmark from the PolyBench benchmark suite shown in Figure 9. The loops $i$ and $j$ cannot be permuted to the outermost level, because it violates the anti-dependencies from $S_{2}$ to $S_{1}$. Therefore, the outermost loop has to correspond to the dimension $t$. At the second level, one can either distribute the statements to gain inner-parallelism or fuse the $i$ (and $j$ ) loops of both the statements to enhance locality, while sacrificing parallelism. The typed fuse approach favors parallelism and distributes the loop nests. This distribution disables diamond tiling [4] in stencils, resulting in performance degradation of $3 \times$ to $5 \times$, due to poor locality. In the rest of this section, we characterize stencil dependence patterns that have tile-wise concurrent start, and our goal is to automatically identify such program segments and perform maximal fusion in those segments using the greedy approach described in Section 3.4.1.

Though time-iterated stencils lack outer parallelism, concurrent start can be enabled with diamond tiling [4], thereby avoiding pipeline start-up and drain phases. Such stencil patterns are local to SCCs and have the following properties: (1) dependencies span the entire iteration space, or equivalently, there does not exist an outer parallel hyperplane, (2) there is a face with concurrent start, and (3) along a hyperplane that is normal to the face with concurrent start, all dependencies are short, i.e., the dependence distances are not parametric. For each SCC $S$, we first check if the SCC has a parallel hyperplane by solving an LP formulation with tiling validity constraints, dependence distance bounding constraints and trivial solution avoiding constraints, with the same objective function as Pluto. If there exists a parallel hyperplane, then in the solution of the LP formulation, $(\vec{u}, w)=(\overrightarrow{0}, 0)$, as shown by Acharya et al. [1], and the SCC is not marked as a stencil. Otherwise, for the SCC $S$, we find the face $\vec{f}$ that allows concurrent start as described by Bondhugula et al. [4]. This is a generalization of the single statement case proposed previously by Krishnamoorthy et al. [22]. This face with concurrent start satisfies the condition $\vec{f} . \vec{d} \geq 1$ for all intra-SCC dependencies. Then, we find a hyperplane $\vec{h}$ that is normal to $\vec{f}$ using the LP formulation:

$$
\begin{array}{ll}
\operatorname{minimize}(w) & \\
\text { subject to : } & \vec{h} \cdot \vec{f}=0, \\
& \forall \vec{d} .-w \leq \vec{h} \cdot \vec{d} \leq w, \\
& 0 \leq w \leq 10 .
\end{array}
$$


The first constraint in the LP formulation ensures that $\vec{h}$ is normal to $\vec{f}$. The constraint $-w \leq \vec{h} . \vec{d} \leq w$, enforces non-parametric upper and lower bounds on dependence distance for each dependence. This constraint can be linearized by the application of Farkas lemma [41]. The last constraint enforces an upper bound on $w$ in the non-negative half space. A solution to the above LP is a hyperplane along which the dependence distances are constant and are bounded by w. Therefore, for a given SCC that does not have an outer parallel hyperplane, if there exists a solution to the LP formulation shown in Equation (8), the SCC is classified as a stencil. A more precise characterization would be to solve the LP formulation in Equation (8) for every hyperplane $\vec{h}$ that is orthogonal to $\vec{f}$, because, with a single LP, Equation (8) classifies an SCC with dependencies $(1,+,-1),(1,-,-1),(1,0,1),(1,0,-1)$ as a stencil. But for the benchmarks that we studied, a single LP formulation shown in Equation (8) was sufficient.

We add a stencil-detection phase, in which, SCCs in the DDG that exhibit stencil dependence patterns are automatically marked and distributed, before the construction of the fusion conflict graph. For each SCC that is marked as a stencil, parallelism preventing edges are not added during FCG construction. Hence, even in the case of typed fusion, we do not distribute multi-statement stencils to enable tile-wise concurrent start via diamond tiling.

\subsection{Complexity Analysis}

In this section, we provide the time complexity of our approach. Without loss of generality, let us assume that every SCC is of dimensionality $m$ in a program $P$. Let $|\mathcal{S}|$ denote the number of SCCs in DDG of $P$. The construction of FCG by Algorithm 3 has $m \times|\mathcal{S}|$ LPs for the addition of permute preventing edges, and $|\mathcal{S}|^{2} \times m^{2}$ LPs for adding inter-SCC edges. Hence the construction of the FCG is of the order of $\Theta\left(|S|{ }^{5} m^{5}\right)$, assuming the average time complexity of solving an LP is $\Theta\left(n^{3}\right)$, where $n$ is the number of variables in the LP. The coloring routine in the clustered approach checks only $m$ vertices to conclude if coloring failed due to a fusion preventing edge or a permute preventing edge. The greedy choice for coloring a vertex is made by looking at all convex successors of a given SCC. Hence the coloring routine has complexity $\Theta\left(|\mathcal{S}|^{2} m^{2}\right)$. Each of these steps might have to be repeated at most $m$ times and hence the complexity of finding a valid permutation is $\Theta\left(|\mathcal{S}|^{5} \mathrm{~m}^{6}\right)$. The number of statements in the program is of the same order as the number of SCCs in the DDG. Therefore, with a valid permutation found in polynomial time, the Pluto-lp-dfp framework finds affine loop transformations in polynomial time. The above discussion also holds for the typed fuse variant, because the same LP formulation and coloring routines are used by Algorithms 3 and 4.

\subsection{Summary}

We implement a variant of typed fusion, called hybrid-fuse, as the default fusion model in Pluto-lp$\mathrm{dfp}$. This fusion model performs typed fusion at outer levels until a parallel hyperplane for an SCC $S$ is found. Then for the inner levels of $S$, max fusion strategy described in Section 3.4 is adopted. The first step in our auto-transformation framework is to identify SCCs that are stencils. SCCs that are marked as stencils are distributed and parallelism preventing edges are not added. Hence, the construction of FCG for these SCCs is analogous to Algorithm 3. Parallelism preventing edges are added for vertices corresponding to dimensions of non-stencil SCCs using Algorithm 4. Convex independent sets are found using the greedy coloring heuristic. FCG is either reconstructed or updated based on whether the coloring heuristic failed due to a permute or fusion preventing edge in the FCG. Once vertices corresponding to a dimension is colored for every SCC, the valid permutation for every statement is updated. After coloring the entire FCG, loop scaling and shifting factors are found for each level of the valid permuation, and then, loop skewing is introduced 
Table 1. Experimental setup

\begin{tabular}{lc}
\hline Processor & Intel Xeon Silver $4110 @ 2.10 \mathrm{GHz}$ based on Skylake-SP microarchitecture \\
Cores & 16 \\
LLC & $11.2 \mathrm{MB}$ \\
Memory & $256 \mathrm{~GB}$ DDR4, 2666 MT/s \\
OS & Centos 7.6 (Linux kernel 3.10.0-957.1.3.el7.x86_64) \\
\hline Compiler & Intel C Compiler 19.0.4 \\
Flags & $-\mathrm{O} 3$-xHost -ansi-alias -ipo -fp-model precise \\
\hline
\end{tabular}

on a per-SCC basis only if it enables tiling. Hyperplanes that enable tile-wise concurrent start are then found for stencils. The loop nest is tiled and intra-tile optimizations are performed to enhance spatial locality and enable vectorization. This pass moves an intra-tile loop in the innermost permutable band that maximizes stride- 0 and stride- 1 accesses according to a cost function [33], to the innermost level. Register reuse in a loop nest is exploited through multi-loop unroll and jam optimization of loops in the AST. Every intra-tile loop of a tiled loop nest is unroll jammed if (1) the loop has temporal reuse, is not the innermost, and traverses a rectangular domain, and (2) unroll jamming does not increase the number of distinct accesses at the innermost level beyond a particular threshold. Finally, we generate OpenMP parallel C code using a polyhedral code generator.

Pluto-lp-dfp relies on Pluto for loop tiling and intra-tile optimizations. However, the transformations found by Pluto-lp-dfp differ from the transformations found by Pluto in the following ways: (1) the fusion heuristic in Pluto is adhoc and does not take parallelism into account; (2) clustering in Pluto-lp-dfp restricts all statements within an SCC to have the same transformation at a given level. Apart from these differences, in some cases, Pluto may perform loop skewing to enable loop fusion, while Pluto-lp-dfp does not. However, these cases are very rare in practice.

\section{EXPERIMENTAL RESULTS}

In this section, we provide the details of our experiments. We implement our fusion models in the Pluto-lp-dfp framework. We use the recent work of Kong et al. [20], which we refer to as PoCC+ [34], and Pluto [33] (version 0.11.4-920) as baselines for comparison. The results that we present in the rest of this section provide insights on the following: (1) impact of our fusion models in conjunction with affine loop transformations on performance, and (2) the overhead of complex loop fusion heuristics on auto-transformation times of the Pluto-lp-dfp framework when compiling programs with hundreds of statements.

\subsection{Experimental Setup}

All our experiments were performed on a 16 core, dual socket ( 8 cores per socket), Intel Xeon Silver $4110 \mathrm{CPU}$ running at $2.10 \mathrm{GHz}$. OpenMP threads in the auto-transformed codes were explicitly pinned to cores 0 to 15 , and thus, SMT was not utilized. We used Intel C Compiler (icc) to compile the codes generated by PoCC+, Pluto and Pluto-lp-dfp. The detailed experimental setup is provided in Table 1. Unroll and jam was enabled in Pluto with the flag --unrolljam and a default unroll factor of 8 was used. PoCC+ already implements unroll jam of loop nests that have a single statement in the innermost loop. PoCC+ and Pluto were compiled with gcc-8. We used GLPK [13] as the ILP solver with both Pluto and Pluto-lp-dfp and PIP [10] as the ILP solver with PoCC+. A default tile size of 32 was used with both Pluto and Pluto-lp-dfp. CLooG [7] was used to generate $\mathrm{C}$ code from transformations that were obtained by both Pluto and Pluto-lp-dfp. 
Fusion models: We implemented the following three fusion models in Pluto-lp-dfp: (1) maxfuse variant that uses clustering and greedy coloring heuristics described in Section 3.4, (2) typedfuse variant that performs parallelism preserving fusion by adding parallelism preventing edges, as discussed in Section 4, and (3) hybrid-fuse variant that performs typed fusion at outer levels and max fusion at inner levels, as described in Section 4.4.

Benchmarks: We evaluated our approach on benchmarks from PolyBench [35] and NAS parallel benchmark suites. The benchmarks from PolyBench have been widely used to evaluate the performance of polyhedral auto-parallelizers. Extra large dataset in PolyBench was used for evaluation. We also evaluated our fusion models on BT, LU, and SP benchmarks of NAS Parallel Benchmark Suite (version 3.1) using class $B$ dataset. In these three benchmarks, we evaluate the autotransformation times of the rhs routine, which has been previously used by Mehta et al. [27] to study the scalability of the Pluto algorithm.

\subsection{Performance Evaluation}

The execution times on 16 cores of all fusion models are listed in Table 2. The second column provides the execution time of codes generated by an older version of Pluto (version 0.11.4-463), that was similar to the version used by Kong et al. [20] for their comparison of PoCC+ with Pluto. The third and the fourth columns present the execution times of the codes generated by PoCC + and the recent version of Pluto (version 0.11.4-920) that includes improved intra-tile optimizations and unroll and jam heuristics. From Columns 2 and 4, we observe that these optimizations result in a performance improvement over the older version of Pluto by a geomean factor of $1.4 \times$ and over $3 \times$ in benchmarks like 2 and $3 \mathrm{~mm}$. This improved version of Pluto is used as the baseline for our comparison. Columns 5-7 correspond to the execution times of max-fuse, typed-fuse, and hybrid-fusion variants implemented in the Pluto-lp-dfp framework. The last two columns give the speedup of the hybrid-fuse variant with respect to PoCC+ and the current version of Pluto. In the case of stencils, Pluto and all our fusion models in Pluto-lp-dfp perform better than PoCC+ because of diamond tiling. Pluto-lp-dfp performs better than Pluto in the case of multi-statement time-iterated stencils (heat-3d, jacobi-2d) due to a better transformation that increases the L1 hit rate. In cholesky, we observe that transformation found by Pluto-lp-dfp improves register reuse by enabling unroll jam of loops. This was not possible with the transformation found by Pluto. In the case of doitgen and durbin benchmarks, we observe a degradation in performance. In doitgen, Pluto-lp-dfp finds a scaled up version of the transformation found by Pluto. Such transformations are found by Pluto-lp-dfp in cases where there exist spurious loop carried dependencies due to updates to a loop private variable. Such spurious dependencies can be removed by incorporating techniques like variable liberalization [28] or live range re-ordering [46]. In the case of durbin, all our fusion variants find a parallel loop at the innermost level. However, this inner parallel loop not only has significant OpenMP synchronization cost but also suffers from load imbalance, because it traverses a triangular domain. However, Pluto finds a transformation in which loop skewing enables loop fusion, resulting in loss of parallelism. Our fusion models were able to match the execution time of Pluto generated code in a single threaded execution. In future, we would like to have a model to identify scenarios where parallelizing a loop results in performance degradation.

\subsection{Impact on Auto-transformation Times}

In Table 3, we detail the impact of our fusion models on auto-transformation times of the Pluto-lp-dfp framework. In particular, our goal was to measure the compile-time overhead of typed and hybrid fusion variants over the max-fuse variant. The second and third columns list the auto-transformation times of PoCC + and Pluto, the fourth, fifth, and sixth columns provide the auto-transformation times of max-fuse, typed-fuse, and hybrid-fuse variants in the Pluto-lp-dfp 
Table 2. Execution Times on 16 Cores (in Seconds)

\begin{tabular}{|c|c|c|c|c|c|c|c|c|c|}
\hline & \multirow{2}{*}{ Benchmark } & \multirow{2}{*}{$\begin{array}{c}\text { Pluto } \\
0.11 .4-463\end{array}$} & \multirow{2}{*}{$\mathrm{PoCC}+$} & \multirow{2}{*}{$\begin{array}{c}\text { Pluto } \\
0.11 .4-920\end{array}$} & \multicolumn{3}{|c|}{ Pluto-lp-dfp } & \multicolumn{2}{|c|}{ Hybrid-fuse speedup } \\
\hline & & & & & $\max$ & typed & hybrid & $\overline{\mathrm{PoCC}+}$ & Pluto \\
\hline \multirow{2}{*}{$\begin{array}{l}\text { Data } \\
\text { mining } \\
\end{array}$} & correlation & 0.521 & 0.332 & 0.398 & 0.276 & 0.394 & 0.396 & 0.84 & 1.01 \\
\hline & covariance & 0.710 & 0.361 & 0.400 & 0.366 & 0.394 & 0.394 & 0.92 & 1.02 \\
\hline \multirow{18}{*}{$\begin{array}{l}\text { Linear } \\
\text { algebra }\end{array}$} & $2 \mathrm{~mm}$ & 0.908 & 0.236 & 0.289 & 0.294 & 0.286 & 0.293 & 0.81 & 0.99 \\
\hline & $3 \mathrm{~mm}$ & 1.732 & 0.390 & 0.400 & 0.402 & 0.400 & 0.405 & 0.96 & 0.99 \\
\hline & $\operatorname{atax}$ & 0.003 & 0.003 & 0.003 & 0.003 & 0.003 & 0.003 & 0.82 & 0.99 \\
\hline & bicg & 0.003 & 0.003 & 0.003 & 0.003 & 0.003 & 0.003 & 0.87 & 1.01 \\
\hline & cholesky & 1.566 & 1.355 & 1.066 & 0.417 & 0.416 & 0.416 & 3.25 & 2.56 \\
\hline & doitgen & 1.106 & - & 0.831 & 1.352 & 1.327 & 1.335 & - & 0.62 \\
\hline & durbin & 0.020 & 0.020 & 0.020 & 0.051 & 0.050 & 0.050 & 0.39 & 0.39 \\
\hline & gemm & 0.400 & 0.255 & 0.207 & 0.182 & 0.281 & 0.181 & 1.41 & 1.14 \\
\hline & gemver & 0.019 & 0.027 & 0.019 & 1.886 & 0.022 & 0.020 & 1.38 & 0.94 \\
\hline & gesummv & 0.007 & 0.005 & 0.006 & 0.007 & 0.007 & 0.007 & 0.78 & 0.96 \\
\hline & gramschmidt & 3.086 & 8.551 & 1.438 & 1.492 & 1.432 & 1.431 & 5.98 & 1.00 \\
\hline & $\mathrm{lu}$ & 1.820 & 7.942 & 1.731 & 2.052 & 2.052 & 2.054 & 3.87 & 0.84 \\
\hline & mvt & 0.015 & 0.016 & 0.017 & 0.014 & 0.014 & 0.014 & 1.13 & 1.20 \\
\hline & symm & 56.03 & 17.38 & 20.09 & 20.09 & 10.61 & 10.64 & 1.64 & 1.89 \\
\hline & syr2k & 1.444 & 2.196 & 1.119 & 1.185 & 1.190 & 1.182 & 1.86 & 0.95 \\
\hline & syrk & 0.940 & 1.481 & 0.690 & 0.554 & 0.595 & 0.554 & 2.67 & 1.24 \\
\hline & trisolv & 0.004 & 0.020 & 0.005 & 0.017 & 0.005 & 0.005 & 4.23 & 1.17 \\
\hline & trmm & 0.149 & 0.323 & 0.127 & 0.183 & 0.103 & 0.115 & 2.82 & 1.11 \\
\hline \multirow[t]{3}{*}{ Medley } & floyd-warshall & 43.71 & 136.2 & 46.86 & 46.35 & 46.34 & 46.19 & 2.95 & 1.01 \\
\hline & fdtd-2d & 2.418 & 8.588 & 1.776 & 1.770 & 1.764 & 1.762 & 4.87 & 1.01 \\
\hline & heat-3d & 14.75 & 10.88 & 8.687 & 4.967 & 4.956 & 4.961 & 2.19 & 1.75 \\
\hline \multirow[t]{4}{*}{ Stencils } & jacobi-1d & $1.7 \mathrm{E}-3$ & 0.018 & $1.3 \mathrm{E}-3$ & $1.7 \mathrm{E}-3$ & $1.3 \mathrm{E}-3$ & $1.3 \mathrm{E}-3$ & 14.1 & 1.02 \\
\hline & jacobi-2d & 3.394 & 5.482 & 2.341 & 1.357 & 1.355 & 1.369 & 4.00 & 1.71 \\
\hline & seidel-2d & 17.20 & 18.32 & 16.65 & 16.65 & 16.65 & 16.65 & 1.10 & 1.00 \\
\hline & bt & 18.90 & - & 18.26 & 577.7 & 17.53 & 16.91 & - & 1.08 \\
\hline \multirow[t]{3}{*}{ NAS } & lu & 75.24 & - & 79.53 & 2359.7 & 93.98 & 93.88 & - & 0.85 \\
\hline & $\mathrm{sp}$ & 38.42 & - & 37.74 & 1100.6 & 34.78 & 34.30 & - & 1.10 \\
\hline & Geomean & & & & & & & 1.8 & 1.07 \\
\hline
\end{tabular}

Cases in which auto-transformation frameworks did not find a transformation in $10 \mathrm{~h}$ or ran out of memory are marked with a "-."

framework, respectively. The last two columns in Table 3 provide the speedups of the hybrid fuse variant in Pluto-lp-dfp over PoCC+ and Pluto with respect to compilation time. The autotransformation times in the case of the Pluto-lp-dfp framework include time taken for identifying stencil dependence patterns in SCCs, FCG construction and coloring time (Algorithms 1 and 2), time taken to find loop scaling and shifting factors, and time taken by the loop skewing phase. PoCC + does not have support for either Gurobi [15] or GLPK, that is required by the Pluto-lp-dfp framework. Hence, to nullify the effects of the ILP solver, we ignore the constraint solving times in PoCC+, whereas, include these in the automatic transformation times for Pluto and Pluto-lp-dfp.

We first observe that the construction of constraints in PoCC + is significantly slower than Plutolp-dfp. The fusion variants in Pluto-lp-dfp are over $10^{4}$ times faster than PoCC + in the correlation benchmark. The hybrid-fuse variant, which is the best performing variant among the rest in terms 
Table 3. Compilation (Automatic Transformation) Times in Seconds

\begin{tabular}{|c|c|c|c|c|c|c|c|c|}
\hline & \multirow{2}{*}{ Benchmark } & \multirow{2}{*}{ PoCC+ } & \multirow{2}{*}{ Pluto } & \multicolumn{3}{|c|}{ Pluto-lp-dfp } & \multicolumn{2}{|c|}{ Hybrid-fuse speedup } \\
\hline & & & & max-fuse & typed-fuse & hybrid-fuse & $\mathrm{PoCC}+$ & Pluto \\
\hline \multirow{2}{*}{$\begin{array}{l}\text { Data } \\
\text { mining }\end{array}$} & correlation & 463.2 & 0.161 & 0.036 & 0.044 & 0.043 & 10798.3 & 3.762 \\
\hline & covariance & 58.09 & 0.033 & 0.017 & 0.019 & 0.018 & 3153.63 & 1.805 \\
\hline \multirow{18}{*}{$\begin{array}{l}\text { Linear } \\
\text { algebra }\end{array}$} & $2 \mathrm{~mm}$ & 15.81 & 0.019 & 0.011 & 0.014 & 0.014 & 1138.32 & 1.339 \\
\hline & $3 \mathrm{~mm}$ & 72.13 & 0.043 & 0.020 & 0.025 & 0.026 & 2823.06 & 1.700 \\
\hline & atax & 2.100 & $5.1 \mathrm{E}-3$ & $4.0 \mathrm{E}-3$ & $5.5 \mathrm{E}-3$ & $5.1 \mathrm{E}-3$ & 408.81 & 0.993 \\
\hline & bicg & 1.763 & $4.1 \mathrm{E}-3$ & $3.6 \mathrm{E}-3$ & $5.0 \mathrm{E}-3$ & $4.7 \mathrm{E}-3$ & 373.14 & 1.031 \\
\hline & cholesky & 5.750 & 0.029 & 0.007 & 0.011 & 0.011 & 518.91 & 2.593 \\
\hline & doitgen & - & 0.023 & 0.013 & 0.015 & 0.015 & - & 1.551 \\
\hline & durbin & 15.81 & 0.049 & 0.011 & 0.014 & 0.014 & 1129.71 & 3.507 \\
\hline & gemm & 1.626 & $4.6 \mathrm{E}-3$ & $4.0 \mathrm{E}-3$ & $5.3 \mathrm{E}-3$ & $5.1 \mathrm{E}-3$ & 321.38 & 0.919 \\
\hline & gemver & 1.636 & $6.7 \mathrm{E}-3$ & $4.0 \mathrm{E}-3$ & $5.6 \mathrm{E}-3$ & $5.4 \mathrm{E}-3$ & 300.97 & 1.247 \\
\hline & gesummv & 2.372 & $5.9 \mathrm{E}-3$ & $4.2 \mathrm{E}-3$ & $5.6 \mathrm{E}-3$ & $5.2 \mathrm{E}-3$ & 455.81 & 1.143 \\
\hline & gramschmidt & 39.81 & 0.065 & 0.016 & 0.019 & 0.019 & 2084.67 & 3.416 \\
\hline & lu & 4.607 & 0.025 & 0.006 & 0.010 & 0.010 & 484.44 & 2.605 \\
\hline & mvt & 0.271 & 0.002 & 0.002 & 0.003 & 0.003 & 93.27 & 0.844 \\
\hline & symm & 19.82 & 0.028 & 0.012 & 0.015 & 0.015 & 1319.82 & 1.843 \\
\hline & syr2k & 1.150 & $3.8 \mathrm{E}-3$ & $3.5 \mathrm{E}-3$ & $4.5 \mathrm{E}-3$ & $4.3 \mathrm{E}-3$ & 267.75 & 0.901 \\
\hline & syrk & 1.131 & $4.0 \mathrm{E}-3$ & $3.6 \mathrm{E}-3$ & $4.5 \mathrm{E}-3$ & $4.4 \mathrm{E}-3$ & 258.05 & 0.905 \\
\hline & trisolv & 0.615 & $3.9 \mathrm{E}-3$ & $2.9 \mathrm{E}-3$ & $3.9 \mathrm{E}-3$ & $3.9 \mathrm{E}-3$ & 157.63 & 1.000 \\
\hline & trmm & 1.811 & $5.6 \mathrm{E}-3$ & $4.9 \mathrm{E}-3$ & $6.4 \mathrm{E}-3$ & $6.2 \mathrm{E}-3$ & 282.12 & 0.880 \\
\hline \multirow[t]{3}{*}{ Medley } & floyd-warshall & 1.982 & 0.017 & 0.018 & 0.022 & 0.022 & 89.30 & 0.763 \\
\hline & fdtd-2d & 20.59 & 0.045 & 0.029 & 0.040 & 0.040 & 508.91 & 1.113 \\
\hline & heat-3d & 63.58 & 0.083 & 0.102 & 0.133 & 0.134 & 476.09 & 0.618 \\
\hline \multirow[t]{4}{*}{ Stencils } & jacobi-1d & 1.096 & 0.009 & 0.008 & 0.012 & 0.012 & 94.50 & 0.771 \\
\hline & jacobi-2d & 7.276 & 0.033 & 0.034 & 0.046 & 0.046 & 156.81 & 0.701 \\
\hline & seidel-2d & 4.217 & 0.012 & 0.018 & 0.021 & 0.021 & 203.97 & 0.598 \\
\hline & bt & - & 297.41 & 3.04 & 3.25 & 3.24 & - & 91.83 \\
\hline \multirow[t]{3}{*}{ NAS } & lu & - & 30.7E3 & 25.53 & 26.29 & 26.20 & - & 1172.7 \\
\hline & $\mathrm{sp}$ & - & 402.68 & 3.17 & 3.38 & 3.39 & - & 118.90 \\
\hline & Geomean & & & & & & 480.1 & 1.269 \\
\hline
\end{tabular}

Cases in which auto-transformation framework did not terminate in $10 \mathrm{~h}$ or ran out of memory are marked with a “-."

of execution time, has a geomean speedup of $461 \times$ over PoCC + . We also observe that max-fuse, typed-fuse and hybrid-fuse variants are faster than Pluto by $1.60 \times, 1.25 \times$, and $1.27 \times$, respectively. Typed-fuse and hybrid-fuse are slower than max-fuse due to the application of Farkas lemma and more LP calls to identify SCCs with stencil dependence patterns. In the case of NAS benchmarks, we observe that PoCC+ did not terminate in over $10 \mathrm{~h}$ for any benchmark. The max-fuse variant of Pluto-lp-dfp is faster than Pluto by a geomean factor of $246 \times$. On this variant, we observe that clustering provides a geomean improvement of $1.47 \times$ in FCG construction time over the unclustered variant, while the time taken by the FCG coloring routine is almost identical in both cases. The typed and hybrid fusion variants are faster than Pluto by a factor of $234 \times$. More importantly, typed fuse and hybrid fuse variants incur a small compile time overhead of $\approx 5.2 \%$ over max-fuse variant, demonstrating that, using the FCG, complex fusion models that efficiently scale to loop nests with a large number of statements can be incorporated in Pluto-lp-dfp. 


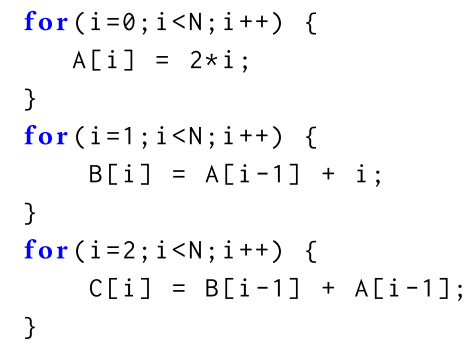

(a) Example code.

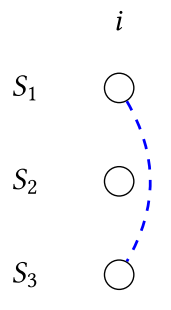

(b) FCG.

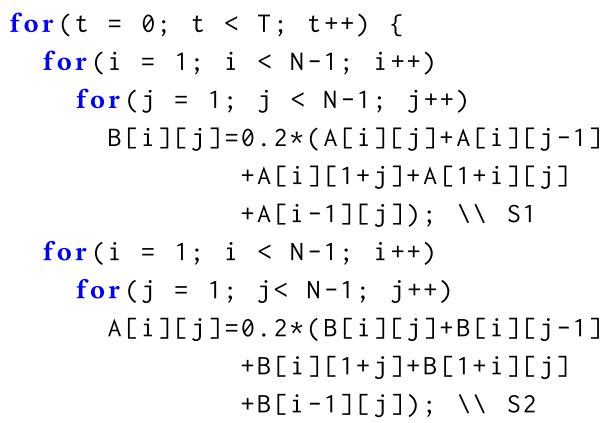

\}

Fig. 9. Typed fusion in stencils.

Fig. 8. Parallelism inhibited by loop shifting.

To summarize, the hybrid-fuse variant in Pluto-lp-dfp framework is faster than Pluto by a geomean factor of $2.2 \times$ in compilation time, with more significant gains on larger NAS benchmarks and over $1,000 \times$ in the largest case. Even when restricted to PolyBench suite, our framework was faster than PoCC + by 461×. These massive improvements in compilation time are meaningful only when there is no loss of perfromance. In terms of execution time, Pluto-lp-dfp outperforms PoCC+ by $1.8 \times$ and an optimized version of Pluto by $1.07 \times$ (maximum of $2.6 \times$ ). This meets our objective of obtaining high performance with low compile time overhead.

\section{RELATED WORK}

Traditionally loop fusion and distribution has been studied in the context of maximizing parallelism and locality. Seminal work by Kennedy and McKinley on parallelism-preserving loop fusion or typed fusion $[18,19]$ involved fusing loops that have the same type (parallel or sequential). Optimal loop fusion has been proven to be NP-complete [19] and Darte [8,9] established NP-completeness for a broader class of loop fusion problems. Both polynomial time and optimal solutions have been proposed for the weighted loop fusion problem [17, 25]. All these approaches do not consider loop fusion in conjunction with other affine loop transformations.

Sarkar and Gao [40] used interference graphs to model loop reversal and loop permutation to enable loop fusion. These graphs have two nodes corresponding to a dimension of a statement. Edges in these graphs are analogous to edges in the FCG. However, their model did not consider transformations that involve loop shifting or loop scaling to enable fusion. Their primary objective was to improve reuse and enable scalarization of arrays and did not consider parallelism in its objective. To model loop reversals in the FCG, we could extend the FCG by duplicating its vertices to model reversals, similar to in interference graphs. However, we observe that, in practice, transformations like loop scaling and loop shifting enable loop fusion in a large number of cases when compared to loop reversals.

Polyhedral compilers like LLVM Polly [14], Pluto, and PPCG [45] model loop distribution with various heuristics. PPCG fuses two SCCs only if it preserves parallelism, while solving more ILP problems. Hence, even with certain heuristics, implementation of parallelism preserving fusion models in a polyhedral compiler would involve solving a significantly large number of ILP formulations, resulting in large auto-transformation times. We also compared the execution times of codes generated by Pluto-lp-dfp and PPCG [37] (which were left out of Section 5 due to space constraints). We observed that Pluto-lp-dfp was faster than PPCG by over 5× (PPCG's heuristics are primarily targeted to GPUs). Pluto distributes SCCs at outermost level based on dimensionalities. At inner levels, loops are distributed only when the ILP formulation is infeasible. This distribution 
is guided by dimensionalities of SCCs, relative positioning of two SCCs in the topological ordering, and when, distribution using either of these heuristics do not satisfy a dependence, all SCCs are distributed at a given level. These heuristics do not consider any costs like loss of parallelism into account. For the example shown in Figure 8, Pluto fuses all the statements resulting in a sequential loop nest.

Mehta et al. [26] provided a loop fusion heuristic in the polyhedral framework called wisefuse, which is similar to typed fusion. Their approach fuses two SCCs only if there is reuse and also ensures that the resulting loop nest remains parallel. Their approach detects if a hyperplane found by Pluto resulted in loss of parallelism and if so, discards the hyperplane found, distributes the SCCs, and then, finds a new hyperplane using Pluto's algorithm. This adds more compile time overhead to the existing auto-transformation times of Pluto (refer to Table 3).

The recent work of Kong and Pouchet [20], which we referred to as PoCC+ in Section 5, obtains significant performance benefits over the then existing version of Pluto (version 0.11.4-85), which we were able to reproduce. They exploit register reuse by unroll and jamming loops, due to which, the benefits of cache tiling diminish. Their loop transformations are guided by a series of objectives based on the characteristics of the program. Experimental results therein concluded that excellent performance improvements could be achieved without actually performing loop tiling. We implemented unroll and jam optimizations in Pluto (and Pluto-lp-dfp) to exploit register reuse and also improved intra-tile optimization heuristics. These optimizations, in addition to loop tiling, not only enabled us to closely match the performance of PoCC + with default tile sizes on benchmarks like 2 and $3 \mathrm{~mm}$ but also achieve overall performance improvement over PoCC + with both Pluto and Pluto-lp-dfp. Tuning for tile sizes will further improve our performance over PoCC+. This re-establishes the need for loop tiling and also motivates further research on tile size selection models. To the best of our knowledge, they are the first to incorporate a model to characterize stencils in a general-purpose compiler. However, their characterization is based on the number of dependencies and the number of statements in the SCoP. Their characterization does not take into account tile-wise concurrent start, constant dependence vectors, absence of communication free parallel loops, which are characteristics of time-iterated stencils, as described in Section 4.2. Finally, the construction of constraints in PoCC + is relatively more expensive, resulting in very high auto-transformation times, as evidenced by our experiments in Section 5.3.

Shirako et al. [42] developed a framework in which polyhedral and AST-based loop transformation approaches work in tandem. Their decoupling is similar to the Pluto-lp-dfp framework and their fusion heuristic is guided by the distinct lines model [39]. They do not consider loop fusion in conjunction with loop scaling. Their approach relies on traditional AST-based methods for loop tiling, while we rely on Pluto, which can tile improper loop nests.

Fusion on coarser grained operators has been widely implemented in domain-specific compilers like Halide [38], XLA [47], and Polymage [32]. Such fusion is more general than traditional loop fusion, since the former could lead to redundant computation and impact intermediary storage in different ways. XLA implements operator fusion on its intermediate representation relying on the type of operators. In Halide and Polymage, stages of an image processing pipeline are fused greedily, based on reuse and the amount of redundant computation [16, 31] among other factors.

\section{CONCLUSIONS AND FUTURE WORK}

We proposed an approach to model loop fusion alongside loop permutations, loop scaling, and loop shifting transformations. We proposed a data structure called the fusion conflict graph and observed that any convex independent set of the fusion conflict graph corresponded to a valid permutation at a given level. Using an SCC-based FCG clustering technique, we implemented a polynomial time greedy coloring heuristic to find convex independent sets. We also proposed 
variants of typed-fusion that fused loops only when they preserved parallelism. We also provided a formulation to identify dependence patterns exhibited by time-iterated stencils and applied fusion heuristics that enabled tile-wise concurrent start for such programs, as part of a single auto-transformation algorithm. In our experiments, the hybrid fusion model outperformed stateof-the-art polyhedral auto-parallelizers like PoCC + and Pluto by mean factors of $1.8 \times$ and $1.07 \times$, respectively, while the compilation time improvement of Pluto-lp-dfp over Pluto was retained.

\section{ACKNOWLEDGMENTS}

We are grateful to the reviewers of TACO for their detailed reviews. We also thank Martin Kong for sharing the implementation of $\mathrm{POCC}+$, which motivated the enhancement of intra-tile and unroll-jam optimizations in Pluto.

\section{REFERENCES}

[1] Aravind Acharya, Uday Bondhugula, and Albert Cohen. 2018. Polyhedral auto-transformation with no integer linear programming. In Proceedings of the 39th ACM SIGPLAN Conference on Programming Language Design and Implementation (PLDI'18). 529-542.

[2] Vinayaka Bandishti, Irshad Pananilath, and Uday Bondhugula. 2012. Tiling stencil computations to maximize parallelism. In Supercomputing. Article 40, 11 pages.

[3] Uday Bondhugula, Aravind Acharya, and Albert Cohen. 2016. The pluto+ algorithm: A practical approach for parallelization and locality optimization of affine loop nests. ACM Trans. Program. Lang. Syst. 38, 3, Article 12 (Apr. 2016), 32 pages.

[4] U. Bondhugula, V. Bandishti, and I. Pananilath. 2017. Diamond tiling: Tiling techniques to maximize parallelism for stencil computations. IEEE Trans. Parallel Distrib. Syst. 28, 5 (May 2017), 1285-1298.

[5] Uday Bondhugula, Muthu Baskaran, Sriram Krishnamoorthy, J. Ramanujam, Atanas Rountev, and P. Sadayappan. 2008. Automatic transformations for communication-minimized parallelization and locality optimization in the polyhedral model. In Proceedings of the foint European Conferences on Theory and Practice of Software 17th International Conference on Compiler Construction (CC'08/ETAPS'08). 132-146.

[6] Uday Bondhugula, Albert Hartono, J. Ramanujam, and P. Sadayappan. 2008. A practical automatic polyhedral parallelizer and locality optimizer. In Proceedings of the 29th ACM SIGPLAN Conference on Programming Language Design and Implementation (PLDI'08). 101-113.

[7] Cloog 2004. The Chunky Loop Generator. Retrieved from http://www.cloog.org.

[8] A. Darte. 1999. On the complexity of loop fusion. In Proceedings of the International Conference on Parallel Architectures and Compilation Techniques (Cat. No. PR00425). 149-157.

[9] Alain Darte. 2000. On the complexity of loop fusion. Parallel Comput. 26, 9 (2000), 1175-1193.

[10] P. Feautrier. 1988. Parametric integer programming. RAIRO Recherche Opérationnelle 22, 3 (1988), 243-268.

[11] P. Feautrier. 1992. Some efficient solutions to the affine scheduling problem: Part I, One-dimensional time. Int. F. Parallel Program. 21, 5 (1992), 313-348.

[12] P. Feautrier. 1992. Some efficient solutions to the affine scheduling problem: Part II, multidimensional time. Int. $\mathcal{F}$. Parallel Program. 21, 6 (1992), 389-420.

[13] GNU. [n.d.]. GLPK (GNU Linear Programming Kit). Retrieved from https://www.gnu.org/software/glpk/.

[14] Tobias Grosser, Hongbin Zheng, Ragesh Aloor, Andreas Simburger, Armin Groslinger, and Louis-Noël Pouchet. 2011. Polly-polyhedral optimization in LLVM. In Proceedings of the 1st International Workshop on Polyhedral Compilation Techniques (IMPACT'11).

[15] Inc. Gurobi Optimization. 2016. Gurobi Optimizer Reference Manual. Retrieved from http://www.gurobi.com.

[16] Abhinav Jangda and Uday Bondhugula. 2018. An effective fusion and tile size model for optimizing image processing pipelines. In Proceedings of the 23rd ACM SIGPLAN Symposium on Principles and Practice of Parallel Programming (PPoPP'18). 261-275.

[17] K. Kennedy. 2000. Fast greedy weighted fusion. In Proceedings of the ACM International Conference on Supercomputing.

[18] K. Kennedy and K. McKinley. 1992. Optimizing for parallelism and data locality. In Proceedings of the ACM International Conference on Supercomputing. 323-334.

[19] K. Kennedy and K. McKinley. 1993. Maximizing loop parallelism and improving data locality via loop fusion and distribution. In Languages and Compilers for Parallel Computing. Springer, 301-320.

[20] Martin Kong and Louis-Noël Pouchet. 2019. Model-driven transformations for multi- and many-core CPUs. In Proceedings of the 40th ACM SIGPLAN Conference on Programming Language Design and Implementation (PLDI'19). 469484. 
[21] Martin Kong, Richard Veras, Kevin Stock, Franz Franchetti, Louis-Noël Pouchet, and P. Sadayappan. 2013. When polyhedral transformations meet SIMD code generation. In Proceedings of the 34th ACM SIGPLAN Conference on Programming Language Design and Implementation (PLDI'13). 127-138.

[22] Sriram Krishnamoorthy, Muthu Baskaran, Uday Bondhugula, J. Ramanujam, Atanas Rountev, and P. Sadayappan. 2007. Effective automatic parallelization of stencil computations. In Proceedings of the 28th ACM SIGPLAN Conference on Programming Language Design and Implementation (PLDI'07). 235-244.

[23] A. Lim and Monica S. Lam. 1998. Maximizing parallelism and minimizing synchronization with affine partitions. Parallel Comput. 24, 3-4 (1998), 445-475.

[24] Amy W. Lim, Gerald I. Cheong, and Monica S. Lam. 1999. An affine partitioning algorithm to maximize parallelism and minimize communication. In Proceedings of the 13th International Conference on Supercomputing (ICS'99). 228-237.

[25] Nimrod Megiddo and Vivek Sarkar. 1997. Optimal weighted loop fusion for parallel programs. In Proceedings of the Ninth Annual ACM Symposium on Parallel Algorithms and Architectures (SPAA'97). 282-291.

[26] Sanyam Mehta, Pei-Hung Lin, and Pen-Chung Yew. 2014. Revisiting loop fusion in the polyhedral framework. In Proceedings of the 19th ACM SIGPLAN Symposium on Principles and Practice of Parallel Programming (PPoPP'14). 233246.

[27] Sanyam Mehta and Pen-Chung Yew. 2015. Improving compiler scalability: Optimizing large programs at small price. In Proceedings of the 36th ACM SIGPLAN Conference on Programming Language Design and Implementation (PLDI'15). 143-152.

[28] Sanyam Mehta and Pen-Chung Yew. 2016. Variable liberalization. ACM Trans. Archit. Code Optim. 13, 3, Article 23 (Aug. 2016), 25 pages.

[29] Benoît Meister, Nicolas Vasilache, David Wohlford, Muthu Baskaran, Allen Leung, and Richard Lethin. 2011. R-stream compiler. In Encyclopedia of Parallel Computing. Springer, 1756-1765.

[30] MLIR 2019. Multi-Level Intermediate Representation Compiler Infrastructure. Retrieved from https://mlir.llvm.org.

[31] Ravi Teja Mullapudi, Andrew Adams, Dillon Sharlet, Jonathan Ragan-Kelley, and Kayvon Fatahalian. 2016. Automatically scheduling halide image processing pipelines. ACM Trans. Graph. 35, 4, Article 83 (July 2016), 11 pages.

[32] Ravi Teja Mullapudi, Vinay Vasista, and Uday Bondhugula. 2015. PolyMage: Automatic optimization for image processing pipelines. In Proceedings of the 20th International Conference on Architectural Support for Programming Languages and Operating Systems (ASPLOS'15). 429-443.

[33] Pluto 2008. PLUTO: A Polyhedral Automatic Parallelizer and Locality Optimizer for Multicores. Retrieved from https://github.com/bondhugula/pluto.

[34] PoCC 2019. The Polyhedral Compiler Collection. Obtained via private communication.

[35] Polybench 2010. Polybench suite. Retrieved from http://polybench.sourceforge.net.

[36] Louis-Noël Pouchet, Uday Bondhugula, Cédric Bastoul, Albert Cohen, J. Ramanujam, P. Sadayappan, and Nicolas Vasilache. 2011. Loop transformations: Convexity, pruning and optimization. In Proceedings of the ACM SIGACTSIGPLAN Symposium on Principles of Programming Languages (POPL'11).

[37] PPCG 2013. Polyhedral Parallel Code Generator. Retrieved from https://github.com/Meinersbur/ppcg.

[38] Jonathan Ragan-Kelley, Connelly Barnes, Andrew Adams, Sylvain Paris, Frédo Durand, and Saman Amarasinghe. 2013. Halide: A language and compiler for optimizing parallelism, locality, and recomputation in image processing pipelines. In Proceedings of the ACM SIGPLAN Conference on Programming Language Design and Implementation (PLDI'13). 519-530.

[39] V. Sarkar. 1997. Automatic selection of high-order transformations in the IBM XL FORTRAN compilers. IBM F. Res. Dev. 41, 3 (May 1997), 233-264.

[40] Vivek Sarkar and Guang R. Gao. 1991. Optimization of array accesses by collective loop transformations. In Proceedings of the 5th International Conference on Supercomputing (ICS'91). 194-205.

[41] Alexander Schrijver. 1986. Theory of Linear and Integer Programming. John Wiley \& Sons.

[42] Jun Shirako, Louis-Noël Pouchet, and Vivek Sarkar. 2014. Oil and water can mix: An integration of polyhedral and AST-based transformations. In Proceedings of the International Conference for High Performance Computing, Networking, Storage, and Analysis (SC'14). 287-298.

[43] Nicolas Vasilache, Benoit Meister, Muthu Baskaran, and Richard Lethin. 2012. Joint scheduling and layout optimization to enable multi-level vectorization. In Proceedings of the International Workshop on Polyhedral Compilation Techniques (IMPACT'12).

[44] Sven Verdoolaege. 2010. ISL: An integer set library for the polyhedral model. In Proceedings of the International Conference on Mathematical Software (ICMS'10), Komei Fukuda, Joris Hoeven, Michael Joswig, and Nobuki Takayama (Eds.). Vol. 6327. Springer, 299-302.

[45] Sven Verdoolaege, Juan Carlos Juega, Albert Cohen, José Ignacio Gómez, Christian Tenllado, and Francky Catthoor. 2013. Polyhedral parallel code generation for CUDA. ACM Trans. Archit. Code Optim. 9, 4, Article 54 (Jan. 2013 ), 23 pages. 
[46] Sven Verdoolaege and Albert Cohen. 2016. Live-range reordering. In Proceedings of the International Workshop on Polyhedral Compilation Techniques (IMPACT'16).

[47] XLA 2017. Optimizing Compiler for Machine Learning. Retrieved from https://www.tensorflow.org/xla.

[48] Oleksandr Zinenko, Sven Verdoolaege, Chandan Reddy, Jun Shirako, Tobias Grosser, Vivek Sarkar, and Albert Cohen. 2018. Modeling the conflicting demands of parallelism and temporal/spatial locality in affine scheduling. In Proceedings of the 27th International Conference on Compiler Construction (CC'18). 3-13.

Received February 2020; revised June 2020; accepted July 2020 